\title{
Sliding Adhesion Confers Coherent Motion to Hair Cell Stereocilia and Parallel Gating to Transduction Channels
}

\author{
K. Domenica Karavitaki ${ }^{1,2}$ and David P. Corey ${ }^{1,2}$ \\ ${ }^{1}$ Department of Neurobiology and ${ }^{2}$ Howard Hughes Medical Institute, Harvard Medical School, Boston, Massachusetts 02115
}

When the tip of a hair bundle is deflected by a sensory stimulus, the stereocilia pivot as a unit, producing a shearing displacement between adjacent tips. It is not clear how stereocilia can stick together laterally but still shear. We used dissociated hair cells from the bullfrog saccule and high-speed video imaging to characterize this sliding adhesion. Movement of individual stereocilia was proportional to height, indicating that stereocilia pivot at their basal insertion points. All stereocilia moved by approximately the same angular deflection, and the same motion was observed at 1,20, and $700 \mathrm{~Hz}$ stimulus frequency. Motions were consistent with a geometric model that assumes the stiffness of lateral links holding stereocilia together is $>1000$ times the pivot stiffness of stereocilia and that these links can slide in the plane of the membrane-in essence, that stereocilia shear without separation. The same motion was observed when bundles were moved perpendicular to the tip links, or when tip links, ankle links, and shaft connectors were cut, ruling out these links as the basis for sliding adhesion. Stereocilia rootlets are angled toward the center of the bundle, tending to push stereocilia tips together for small deflections. However, stereocilia remained cohesive for deflections of up to $\pm 35^{\circ}$, ruling out rootlet prestressing as the basis for sliding adhesion. These observations suggest that horizontal top connectors mediate a sliding adhesion. They also indicate that all transduction channels of a hair cell are mechanically in parallel, an arrangement that may enhance amplification in the inner ear.

\section{Introduction}

The mechanosensitive stereocilia extending from the apical surface of a hair cell form a compact bundle with the tips of stereocilia in close apposition (see Fig. $1 A$ ). Arranged in a graded series of heights, stereocilia are connected in columns along the shortto-tall axis by tip links that extend upward from the tip of each stereocilium to the side of its taller neighbor. Deflection toward the tallest stereocilia causes shearing between adjacent stereocilia of each column that increases tip link tension and pulls open force-gated transduction channels.

Exactly what forces are felt by different transduction channels during deflection, and how the opening of one channel affects forces on the others, are not well characterized. They depend critically on the extent to which stereocilia remain cohesive as the bundle is deflected, and on what holds stereocilia together. Adjacent stereocilia are connected by tip links, by horizontal top connectors located near stereocilia tips, by shaft connectors distributed more broadly along the lengths of stereocilia, and by ankle links near the basal tapers, although the density and number of links varies among species, inner-ear organ, and developmental stage (Nayak et al., 2007). It is unclear which contribute to bundle cohesion.

One possibility is that tip links provide the main connection between stereocilia. If so, the transduction channels of a column

\footnotetext{
Received Sept. 30, 2009; revised April 28, 2010; accepted May 17, 2010.

This work was supported by National Institutes of Health Grant R01DC000304 (D.P.C.). D.P.C. is an Investigator and K.D.K. was an Associate of the Howard Hughes Medical Institute. We appreciate help from Dr. Bernardo Sabatin with two-photon imaging and from Dr. Elio Raviola with electron microscopy.

Correspondence should be addressed to K. Domenica Karavitaki, Department of Neurobiology, Harvard Medical School, 220 Longwood Avenue, Boston, MA 02115. E-mail: domenica@alum.mit.edu.

D0I:10.1523/JNEUROSCI.4864-09.2010

Copyright $\odot 2010$ the authors $\quad 0270-6474 / 10 / 309051-13 \$ 15.00 / 0$
}

would be mechanically in series, and the opening of one channel would reduce the force on others, producing a negative cooperativity. In addition, if forces are delivered to the tallest stereocilia (which reflects the normal coupling in vivo), the stretch of elastic components of the transduction apparatus would allow separation or splay between adjacent stereocilia. Alternatively, if stereocilia are strongly held together by other connectors or adhesive mechanisms, then the bundle should move as a unit without splay, and all transduction channels of a cell would be mechanically in parallel (Karavitaki and Corey, 2005).

Visual and video observations of hair bundle motion during slow $(\sim 1 \mathrm{~Hz})$ deflections suggested that bundles of bullfrog saccular hair cells and turtle cochlear hair cells move as a unit without splay (Hudspeth, 1983; Crawford and Fettiplace, 1985; Corey et al., 1989; Assad and Corey, 1992). Recently, optical measurements confirmed that the bundle moves as a unit even up to high frequencies (Karavitaki and Corey, 2005; Kozlov et al., 2007), at least for small deflections ( $<50 \mathrm{~nm})$. However, video measurements of chick hair cells (Duncan et al., 1995) suggested that stereocilia do not move uniformly, and some modeling efforts have assumed that only tip links hold stereocilia together (Nam et al., 2006, 2007a,b).

Here, we use high-resolution video microscopy to show that bullfrog stereocilia separation does not increase by more than $\sim 5$ $\mathrm{nm}$ during physiological stimuli $(<300 \mathrm{~nm})$. Cutting ankle links, shaft connectors, and tip links does not allow separation. We suggest that the horizontal top connectors mediate sliding adhesion and thus confer parallel gating to the transduction channels.

\section{Materials and Methods}

Isolated frog hair cells-differential interference contrast microscopy. Isolated hair cells from the bullfrog saccule were dissociated as described previously (Assad et al., 1991). For all experiments, male and female 
bullfrogs were selected randomly. Briefly, saccules were surgically exposed and bathed for $\sim 15 \mathrm{~min}$ in oxygenated perilymph-like solution (120 mм NaCl, 2 mм KCl, 0.1 mм CaCl , 3 mм dextrose, 5 mм HEPES, $\mathrm{pH} \sim 7.3$; final osmolarity, 230-250 mOsm; this medium will be subsequently abbreviated as LCR for low calcium Ringer's) containing $1 \mathrm{~mm}$ EGTA and $1 \mathrm{~mm} \mathrm{MgCl} 2\left(\sim 10^{-8} \mathrm{M}\right.$ free $\mathrm{Ca}^{2+}$ concentration estimated using the Max Chelator program; http://maxchelator.stanford.edu/ CaMgATPEGTA-TS.htm). Saccules were subsequently removed, transferred to a Petri dish containing LCR, and opened before otoconia were dissected away. The otolithic membrane was removed after treating the saccules with $50-75 \mu \mathrm{g} / \mathrm{ml}$ proteinase XXIV (Subtilisin Carlsberg; P8038; Sigma-Aldrich) for 22-28 min. Hair cells were flicked out of the saccule using an eyelash and allowed to settle onto the clean glass bottom of a recording chamber containing LCR solution. In some cases, $10 \mu \mathrm{M}$ FM1-43 (70020; Biotium) or AM1-43 (70024; Biotium) was applied to assess the integrity of transduction by dye accumulation. In some experiments, after identifying the cell of interest, we puffed a perilymph-like solution containing 5 mM BAPTA (B-1214; Invitrogen) onto the hair cell to cut tip links. We allowed the cells to sit in this medium for 2-3 min and then applied $10 \mu \mathrm{M}$ FM1-43 or AM1-43 to assess transduction. We recorded from cells within $3 \mathrm{~h}$ after animal decapitation; cells that had swollen or granulated somas or broken stereocilia were not used.

For video imaging, we selected hair cells that had settled on their sides so that the excitation axis was parallel to the image plane. A hair bundle was displaced using a glass pipette attached by suction to the kinociliary bulb and driven by a piezoelectric bimorph (Corey and Hudspeth, 1980). For cells missing the kinocilium, the stimulus pipette was attached by suction to the tallest stereocilia. Stimuli were sinusoids with frequencies of 1,20 , or $700 \mathrm{~Hz}$ and peak amplitude ranging from 0.25 to $5 \mu \mathrm{m}$. Hair cells were visualized with a $63 \times$ water-immersion objective (Zeiss; $63 \times 1$ $0.9 \mathrm{~W}$; Achroplan, $\infty / 0$ ) with an additional $5 \times$ magnification and (at 1 $\mathrm{Hz}$ ) differential interference contrast (DIC) optics. The resolution of the images was $\sim 30 \mathrm{~nm} /$ pixel. Illumination was a high-power light-emitting diode (LED) (Luxeon; $5 \mathrm{~W}$ Star; $\lambda \sim 505 \mathrm{~nm}$; on-time $<100 \mathrm{~ns}$ ) positioned at the field diaphragm. A CCD camera (Hamamatsu; C2400) was mounted on the phototube of the microscope (Zeiss; Axioskop) and was connected to an image processor (Argus-20; Hamamatsu). An externally triggered frame grabber (AG-5; Scion Corporation) was used for realtime frame capture.

Low-frequency $(1 \mathrm{~Hz}$ ) hair bundle motion was captured at $\sim 11$ frames/s by running the frame grabber on continuous capture. Hair bundle motion at 20 and $700 \mathrm{~Hz}$ was captured by strobing the LED at one of eight equally spaced phases during the stimulus period. For these frequencies, we also collected images with the stimulus turned off, so that before each new phase of motion we acquired a corresponding "nostimulus" phase. Usually only two phases of motion, occurring near the peak stimulus displacement in the excitatory and the inhibitory direction, were analyzed. Acquisition programs were written in LabView 6.1 (National Instruments) and generated the voltages that controlled the bimorph and the current source driving the LED, via a National Instruments A/D board. Resulting images (see Fig. $1 A$ ) were used to create animations of the observed motion. The first steps of image processing were performed with software written using Scion Image's macro programming environment. Images were first smoothed to reduce noise by applying two iterations of a $3 \times 3$ pixel median filter, and one iteration of a $3 \times 3$ pixel smoothing filter whose center weighting factor was four times that of the surrounding pixels. Regions of interest within an image (such as the edges of individual stereocilia) were selected. The next steps of image processing and motion analysis were performed with software written using the MATLAB platform. The selected images (usually $16 \times$ 16 pixel squares) were interpolated and, depending on the contrast of the image, high-pass filtered as described previously (Karavitaki and Mountain, 2007). For the $1 \mathrm{~Hz}$ stimulus frequency, cross-correlation methods were used to quantify the motion of the region of interest between the two extreme phases of motion. For the higher frequencies, crosscorrelation was performed between the excitatory or inhibitory phase of motion and the corresponding no-stimulus image.

For "combing" experiments, hair cells were isolated as described above. A pipette of $\sim 50 \mathrm{~nm}$ tip diameter, pulled and bent as described previously (Hudspeth and Corey, 1978), was manipulated between adjacent stereocilia at their bases and moved along their length. Depending on the experiment (see Results), either the combing pipette or a separate pipette attached by suction to the kinociliary bulb was used to deflect the hair bundle. Throughout these experiments, the cells were imaged under DIC optics and were recorded using the frame grabber set on continuous capture.

Intact saccular epithelia-two-photon microscopy. Saccules were removed from a bullfrog and placed in oxygenated LCR solution. For some experiments, D-sorbitol was added to increase the osmolarity of the medium (to $\sim 330 \mathrm{mOsm}$ ) without affecting its ionic strength as hyperosmotic solutions have been found to retard bleb formation (Shi et al., 2005). After otoconia were removed, saccules were treated with 50-75 $\mu \mathrm{g} / \mathrm{ml}$ proteinase XXIV (Subtilisin Carlsberg; P8038; Sigma-Aldrich) for $\sim 28 \mathrm{~min}$ to remove their otolithic membranes. They were then pinned flat using a pair of metal pins (item no. 26002-10; Fine Science Tools) that were mounted with Sylgard on a $12 \mathrm{~mm}$ round glass coverslip (Thermo Fisher Scientific) and transferred to the stage of a custom two-photon laser-scanning microscope (Carter and Sabatini, 2004) with an $840 \mathrm{~nm}$ excitation wavelength. The epithelia were then bathed in a solution containing $10 \mu \mathrm{M}$ FM1-43.

The hair bundles were displaced using a glass pipette that was attached by suction to the kinocilium (as visualized under DIC optics) and driven by a piezoelectric bimorph at $\sim 1 \mathrm{~Hz}$. Pockel cells controlled the duration and timing of the two-photon laser illumination, to elicit fluorescence at one of eight phases along the stimulus cycle. Four $32 \times 256$ images for each phase were averaged. Images spaced $0.5-1 \mu \mathrm{m}$ apart along the $z$-axis were collected to cover the entire height of the hair bundles. Images were acquired with a $60 \times$ water-immersion lens (Zeiss; $60 \times 10.9$; LumPlanFI/ IR). Acquisition software was written in MATLAB (Carter and Sabatini, 2004). Off-line data analysis was performed using custom algorithms written in MATLAB and Excel.

Transmission electron microscopy. Three dissection protocols were used before fixation. For one set of experiments, saccules were dissected as described for the intact saccular epithelia experiments and enzymatically treated as before to remove the otolithic membrane. For a second set, no enzymatic treatment was used and the otolithic membrane was left intact. For a third, no enzyme was used but the otolithic membrane was removed with forceps. The saccules were then fixed for $1 \mathrm{~h}$ in $2.5 \%$ glutaraldehyde in $0.1 \mathrm{~m}$ sodium cacodylate buffer, $\mathrm{pH} 7.2$, with $1 \%(\mathrm{w} / \mathrm{v})$ tannic acid. After rinsing three times ( $\sim 5 \mathrm{~min}$ each) in $0.1 \mathrm{~m}$ sodium cacodylate buffer, saccules were postfixed in $1 \%$ osmium tetroxide in 0.1 $\mathrm{M}$ sodium cacodylate buffer for $2 \mathrm{~h}$ and rinsed briefly in water. The samples were dehydrated through 50,70, 90, and 100\% ethanol (one time for 5 min each; all dilutions with distilled water) and 100\% ethanol (two times for $10 \mathrm{~min}$ each). Ethanol was then replaced with two changes of propylene oxide (15 min each), and saccules were immersed in a 1:1 mixture of TAAB 812 resin and propylene oxide overnight. The saccules were then placed in pure resin in flat embedding molds, allowed to sit at room temperature for $1 \mathrm{~h}$, and then cured at $60^{\circ} \mathrm{C}$ for $24-48 \mathrm{~h}$. Throughout the fixation protocol, all saccule transfers were performed with custom-made glass bowls to ensure that the hair bundles were not disrupted by passage through a meniscus.

Thin sections $(50-80 \mathrm{~nm})$ oriented parallel to the long axis of the hair cells were cut using a Reichert Ultracut $S$ microtome, placed on EM grids, and stained with $1.4 \%$ lead citrate. The sections were viewed with a Tecnai $\mathrm{G}^{2}$ Spirit BioTWIN electron microscope at an accelerating voltage of $80 \mathrm{kV}$.

Chick utricle hair cells. Utricular maculae were dissected from embryonic day 16 (E16) to E20 randomly selected male and female chicks, and the otolithic membranes were removed. Dissection was done in Medium 199 (Invitrogen; 11043) supplemented with 5 mM HEPES, or in Medium 199 (Invitrogen; 12340; which contains 25 mM HEPES). For some experiments the epithelia were treated for $22 \mathrm{~min}$ in $50 \mu \mathrm{g} / \mathrm{ml}$ proteinase XXIV (Subtilisin Carlsberg; P8038; Sigma-Aldrich) and subsequently for 20 min in 5 mm BAPTA to remove the ankle links, shaft connectors, and tip links (Bashtanov et al., 2004). Epithelia were transferred to a recording dish, folded in half along the medial/lateral axis so the optical axis was perpendicular to hair bundles (Goodyear and Richardson, 1992), and held under a metal pin. Recordings were made in the middle extrastriolar 
region at the edge of the folded epithelium. Hair bundles were displaced with a glass pipette attached by suction to the tallest stereocilium, using a micromanipulator (MPC-385-2; Sutter Instrument). Hair cells were visualized with a $63 \times$ water-immersion objective (Zeiss; $63 \times / 0.9 \mathrm{~W}$; Achroplan, $\infty / 0$ ) with an additional $2.5 \times$ magnification and DIC optics. Resolution at the camera was $\sim 60 \mathrm{~nm} /$ pixel.

Solutions used to test charge interactions. To test the effect of polycations, we bathed the isolated cells in LCR that also contained $100 \mathrm{~mm}$ $\mathrm{La}^{3+}$. A low ionic strength solution was prepared from $3 \mathrm{~mm}$ dextrose, 5 mM HEPES, 244 mm D-sorbitol, pH 7.28 adjusted with $\mathrm{NaOH}$. Our high ionic strength solution had either 2,3 , or $5 \mathrm{M} \mathrm{NaCl}$ and $2 \mathrm{~mm} \mathrm{KCl}, 0.1 \mathrm{~mm}$ $\mathrm{CaCl}_{2}, 3 \mathrm{~mm}$ dextrose, $5 \mathrm{~mm}$ HEPES, $\mathrm{pH} \sim 7.3$. We also prepared control media that had the same osmolarity as the $5 \mathrm{M} \mathrm{NaCl}$ solution. This control medium was LCR with the addition of $10 \mathrm{M}$ D-sorbitol. To test the effect of $\mathrm{pH}$, we adjusted the $\mathrm{pH}$ of LCR using either $\mathrm{NaOH}$ (for high $\mathrm{pH}$ solutions) or $\mathrm{HCl}$ (for low $\mathrm{pH}$ solutions).

\section{Results}

To understand the movement of stereocilia in a hair bundle, we dissociated hair cells from the bullfrog saccule. Dissociated cells settled to the glass bottom of an experimental chamber and adhered to the clean glass. We chose cells that settled so the excitatory axis of the bundle was parallel to the glass and observed them with high-resolution DIC optics. Images were acquired with a cooled CCD camera and recorded to disk. A stimulus probe, attached by suction to the bulb of the kinocilium (or occasionally to the tallest stereocilia), was moved along the excitatory axis by a piezoelectric bimorph element.

\section{How closely do stereocilia remain together during bundle deflection? \\ Visual assessment}

Deflection of the kinocilium of a bundle in the positive or negative direction caused the bundle to move as a unit. Stereocilia appeared to pivot at their bases, and stereocilia did not separate perceptibly even for deflections of $1 \mu \mathrm{m}\left(7^{\circ}\right)$ or more. (See supplemental movies, available at www.jneurosci.org as supplemental material.) Similar results have been reported for several species (Flock et al., 1977; Hudspeth, 1983; Crawford and Fettiplace, 1985; Howard et al., 1988; Corey et al., 1989; Crawford et al., 1989; Jacobs and Hudspeth, 1990; Karavitaki and Corey, 2005; Kozlov et al., 2007).

\section{Bending}

To quantify movement, the kinocilium was moved by $\pm 0.5 \mu \mathrm{m}$ at a frequency of $\sim 1 \mathrm{~Hz}$, and DIC images representing the peak displacements were acquired. We used an image-processing algorithm to measure the deflection of several stereocilia within a bundle, at 10-40 locations along their lengths (Fig. 1A). The magnitude of displacement at different locations was plotted relative to height. Although we could not determine from the DIC image the actual point of insertion of a stereocilium into the cuticular plate, for each stereocilium, we calculated an effective pivot point, by extrapolating down to zero displacement; this pivot point was used in all further calculations. The movement of the measured points was linear with height (Fig. $1 B$ ), consistent with an absence of bending along the shaft of the stereocilium. Results were similar for different stereocilia within a hair bundle (Fig. 1C,D). We tried fitting curved lines to the data to represent bending, but the fit was not significantly better than for a straight line. Thus, it appears that all bending of a stereocilium during deflection takes place in the lowest micrometer, most likely at the narrow insertion point.
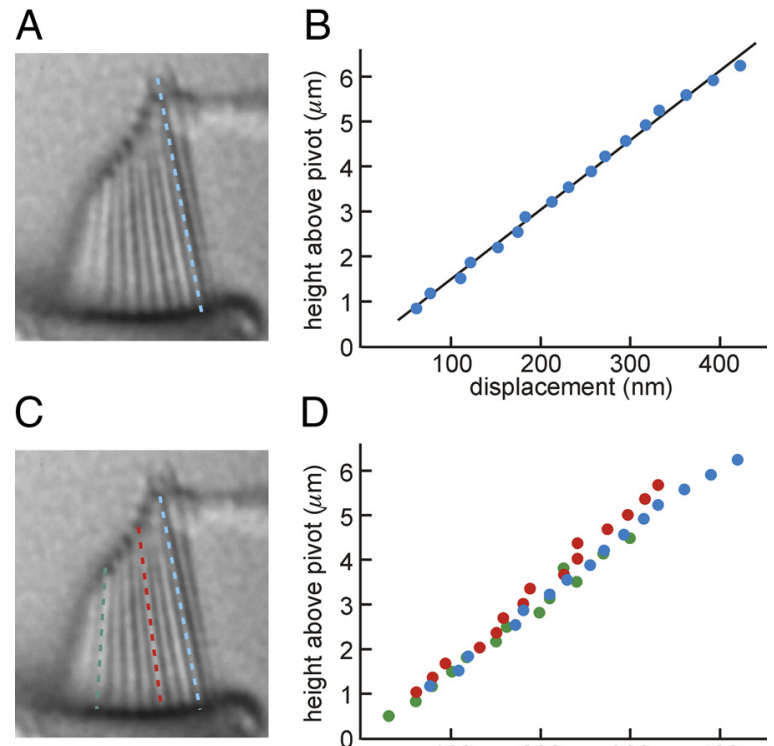

D
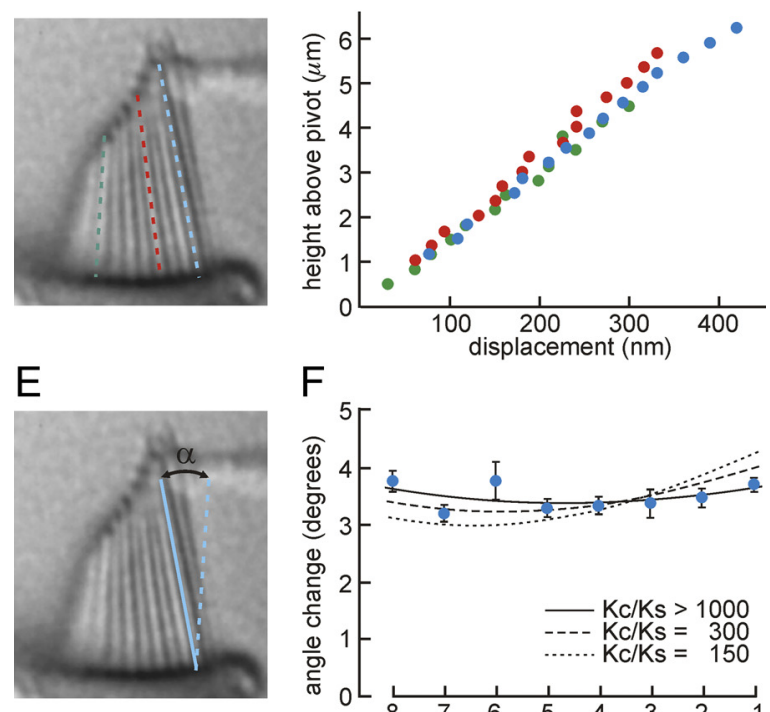

F
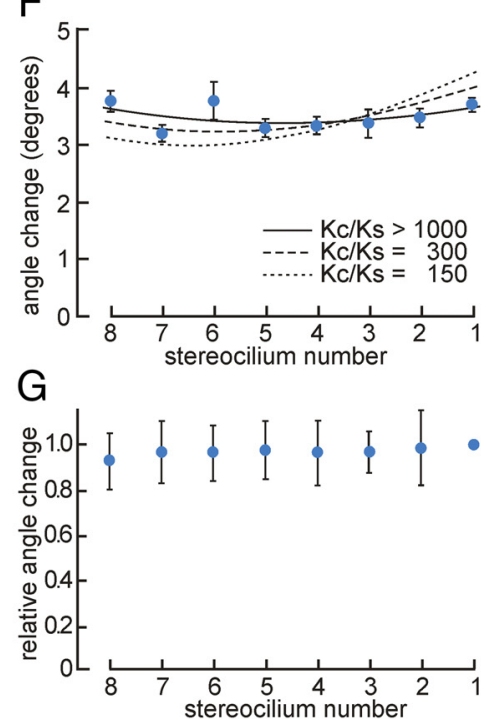

Figure 1. Hair bundle motion at low frequency $(1 \mathrm{~Hz}) . A$, DIC image of a bullfrog saccular hair cell showing the glass probe attached to the kinociliary bulb. The dashed line indicates the tallest stereocilium (no. 1) in the column. $\boldsymbol{B}$, Peak excitatory displacement of the tallest stereocilium for 17 locations along the length of the stereocilium. $C, D$, Peak displacement of three stereocilia in the same column (indicated by the dashed lines). $\boldsymbol{E}, \boldsymbol{F}$, Angular deflection of all stereocilia in the same column. The mean and SD were calculated from all the extractions along the length of individual stereocilia. The solid line indicates predictions of a geometric model that assumes no splay (touch model; $K_{\mathrm{c}} K_{\mathrm{s}}>1000$, where $K_{\mathrm{c}}$ indicates coupling stiffness, and $K_{\mathrm{s}}$ indicates pivot stiffness; $\left.\chi^{2}=6.4\right)$; the dashed lines indicate models with some splay $\left(K_{c} / K_{s}=\right.$ $300, \chi^{2}=15.6 ;$ or $\left.K_{c} / K_{s}=150, \chi^{2}=40.1\right)$. Goodness of fit was estimated by computing the modified $\chi^{2}$ test statistic, taking into account the SD of the data. $\mathbf{G}$, Angular displacement from nine cells. Data are normalized to the angular displacement of the tallest stereocilium for each experiment. The mean and SD were calculated from the normalized averaged data from all experiments.

\section{Separation or splay}

How much the stereocilia of a bundle separate during positive deflection has important implications both for how force is transmitted to transduction channels and for understanding what holds the stereocilia together. Figure $1 D$ shows that stereocilia of different heights move by about the same amount during deflection; that is, they have about the same angular deflection. We calculated the angular deflection (indicated in Fig. $1 E$ ) from the 
measured displacements and plotted the deflections for each stereocilium along a column (Fig. $1 F$ ). If there was separation or splay of the bundle, the shorter stereocilia would have smaller angular deflection. For these small deflections $( \pm 0.5 \mu \mathrm{m})$, the shortest moved by about the same angle as the tallest (Fig. $1 F$ ). Figure $1 G$ shows the average angular deflection of each stereocilium relative to the tallest stereocilium for nine experiments using similar maximum deflections (less than $\pm 0.5 \mu \mathrm{m}$ ). All stereocilia moved with the same angular deflection, within the limits of the measurement.

If stereocilia remain touching as the bundle moves, then adjacent stereocilia move by the same amount at their point of contact and-at least as a first approximation-the angular deflection should be the same. To take into account additional factors such as the thickness of a stereocilium and the curvature of the cuticular plate, we developed a simple geometrical model of the hair bundle (Corey et al., 1989; Jacobs and Hudspeth, 1990; Geisler, 1993; Pickles, 1993; Duncan et al., 1999). For each hair bundle, dimensions such as height and thickness of stereocilia, rest angle of the tallest stereocilium, and the distribution of calculated pivot points were used to predict the angular deflection if stereocilia of a column remain in contact at all times (touch model) (see supplemental material, available at www.jneurosci. org). For most bundles with deflections of a few degrees, the tallest and shortest stereocilia are predicted to move by about the same angle, and middle stereocilia slightly less. The measured deflections match the predictions quite well (Fig. $1 F$ ). [For very large deflections, the shortest stereocilia are predicted to move $20-25 \%$ less than the tallest, which was observed (see below).]

We then modified the model to calculate deflections with the assumption that the links that couple stereocilia together-whatever they are-are elastic and allow stereocilia to separate during positive deflections (splay model) (see supplemental material, available at www.jneurosci.org). Links were posited to exert tension approximately perpendicular to a stereocilium but not along its axis; that is, in the model the attachment points of the links could slide as deflection produced shear between adjacent stereocilia. Because attachment points are allowed to slide, very stiff links would prevent separation but would not impede deflection and so would not contribute to overall bundle stiffness. The lines in Figure $1 F$ also show predictions if the link has a coupling stiffness $K_{\mathrm{c}}$ equivalent to 300 or 150 times the pivot stiffness $K_{\mathrm{s}}$ for a stereocilium at that height. These fits are worse than if the coupling stiffness is much higher $(>1000 \times)$ than the pivot stiffness.

This calculation considered only a single column of stereocilia of a bundle. In fact, there are fewer tall or short stereocilia in a frog saccular bundle than those of medium height, so we might expect more splay for the taller (as a few tall stereocilia must pull on many) and less splay for the shorter (as many pull on the shortest few). When we adjusted the model to account for this distribution of heights, the results were not significantly different (data not shown).

We then asked whether these results hold for larger stimuli, including those many times the physiological range. Figure $2 \mathrm{~A}$ shows the relative angular deflections for stereocilia of a column, for positive deflections up to $1.8 \mu \mathrm{m}\left(15^{\circ}\right)$ from rest. Even for the largest deflections, the geometrical model that assumes no separation (touch model) provides a good fit, and models that allow coupling stiffness of only 700 or 300 times the pivot stiffness (splay model) are less good. In cases in which the shortest stereocilia moved through a smaller angle than the tallest, which might suggest splay, the divergence was predicted from the individual dimensions and rest angles of those bundles (listed in sup-
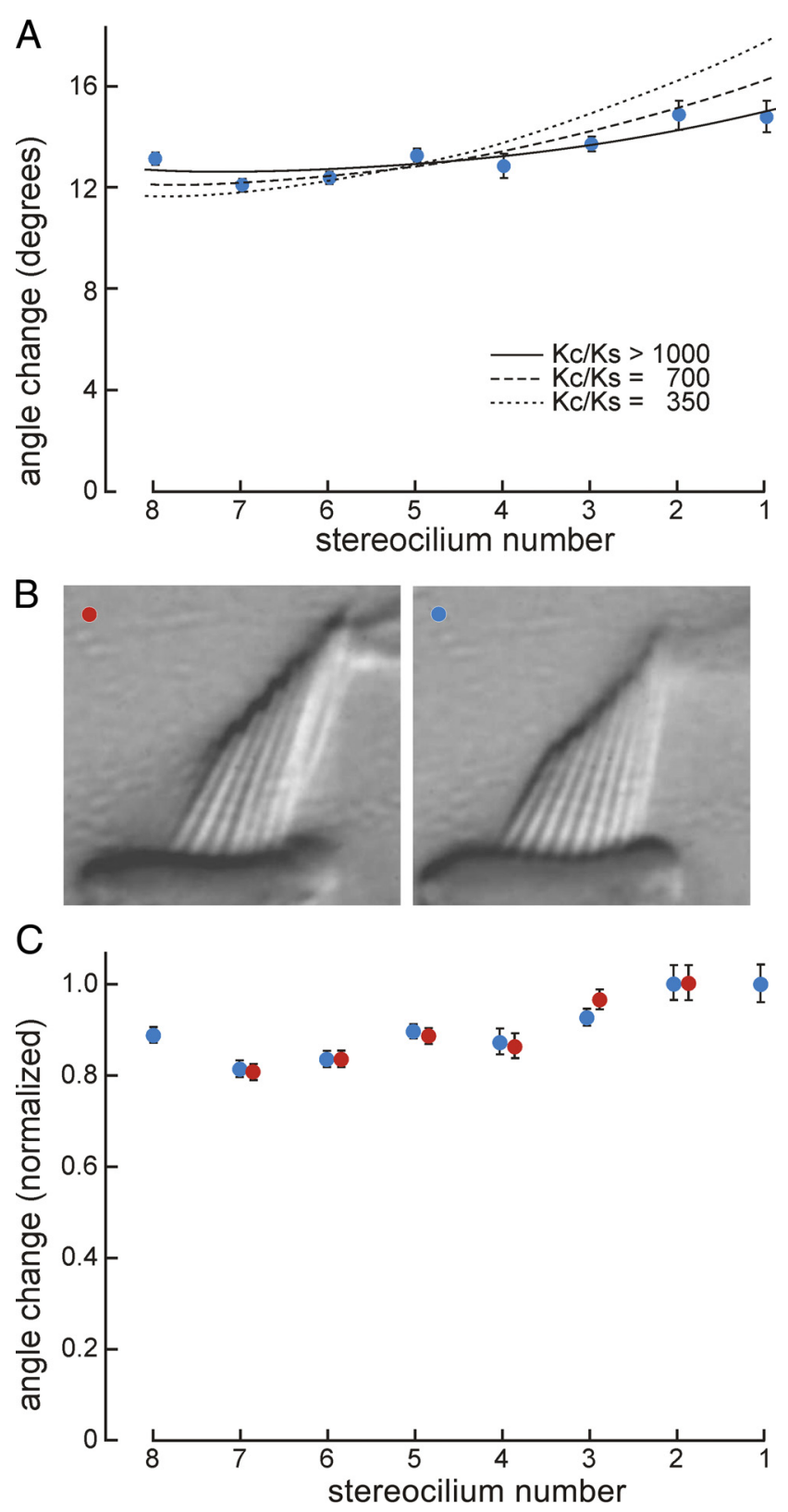

Figure 2. Hair bundle motion for large stimuli at low frequencies. $A$, Angular deflection of each stereocilium along a hair bundle column, for a tip deflection of $1.8 \mu \mathrm{m}$ from rest (mean and SD calculated from all the extractions along the length of each stereocilium). The solid lines are predictions of the touch model $\left(K_{\mathrm{c}} / K_{\mathrm{s}}>1000, \chi^{2}=15.5\right)$; the dashed lines indicate models with some splay $\left(K_{c} / K_{s}=700, \chi^{2}=32.3\right)$, or $K_{c} / K_{s}=350\left(\chi^{2}=89\right)$. Goodness of fit was estimated by computing the modified $\chi^{2}$ test statistic, taking into account the SD of the data. $\boldsymbol{B}$, DIC images of the hair bundle at two focal planes. $C$, Angular deflection of each stereocilium in each of two columns of the bundle, from the two image planes in $\boldsymbol{B}$ (colors as in $\boldsymbol{B}$; data from $\boldsymbol{A}$ replotted). For all panels, stereocilium no. 1 is the tallest.

plemental material, available at www.jneurosci.org) and was inconsistent with separation.

From plots like these, we can calculate that the stiffness of an elastic element holding together a pair of stereocilia is at least 1000 times greater than the pivot stiffness or, alternatively, that the separation between pairs of stereocilia in a column does not increase by $>5 \mathrm{~nm}$ for a deflection equivalent to the operating range of the cell of $\sim 300 \mathrm{~nm}$.

We also asked whether these results hold for columns of stereocilia further away from the axis of symmetry of the hair bun- 
dle. For the same experiment shown in Figure $2 A$ (cell image shown in Fig. $2 B$, blue dot), we focused up closer to the edge of the bundle (cell image shown in Fig. $2 B$, red dot). The angular displacement was similar for both focal depths (Fig. 2C).

\section{Two-photon imaging}

DIC microscopy constructs an image by the interference between two polarized beams passing close together through a sample. In principle, it cuts an optical section through a tissue; however, the DIC image of a bundle could be created by a more complex interference pattern involving many stereocilia and therefore it does not represent a single column. To measure the movement of stereocilia with an independent method, we used two-photon microscopy to image hair bundles in an intact saccular epithelium. The epithelia were bathed in a medium containing dilute FM1-43, a fluorescent styryl dye that partitions into extracellular membranes and thereby becomes fluorescent (Meyers et al., 2003). FM1-43 also passes through open transduction channels, gets trapped within hair cells (Gale et al., 2001; Meyers et al., 2003), and can label the intracellular leaflet of the plasma membranes as well as membranous organelles of the hair cell. Intact transduction was not needed for labeling stereocilia if dye remained in the bath as it partitioned into the outer leaflet of plasma membranes. We viewed hair bundles from the top, cutting optical cross sections parallel to the apical surface of a cell. As before, hair bundles were moved with a stiff probe at $\sim 1 \mathrm{~Hz}$. Individual stereocilia were distinguishable when we focused near the base of the hair bundle, but not when we focused near the apex (Fig. $3 A-D$ ). Consequently, we compared movement of the tallest versus the shortest stereocilia, detected at the edge of the bundle, to measure the degree of separation. Movement of the tallest stereocilia was plotted relative to movement of the shortest, for different heights along a bundle. The relationship followed the unity line (Fig. $3 E$ ), whereas we would expect the points to lie above the unity line if the bundle separated. Within experimental error, no such splay was observed.

These results are in agreement with those obtained using DIC imaging of dissociated hair cells and give us confidence that DIC imaging gives an accurate representation of the stereocilia of a single column.

\section{High-frequency stimuli}

The deflections described above were presented slowly, at $\sim 1 \mathrm{~Hz}$. If stereocilia have a velocity-dependent drag or viscosity, then they might separate at higher frequencies that are beyond the resolution of the video camera. We used a pulsed high-intensity LED light source to produce strobe illumination, delivering a flash at eight points during a sinusoidal stimulus (Fig. $4 \mathrm{~A}$ ). Stimuli were short bursts of 20 and $700 \mathrm{~Hz}$ deflections, a bit below and substantially above the best frequency for the bullfrog saccule, of $\sim 0.5 \mu \mathrm{m}$ peak amplitude. To calculate the motion of stereocilia, we used DIC images only from the two extremes of the stimulus cycle, where the bundle was deflected maximally in the excitatory or the inhibitory direction. Even at higher frequencies (Fig. $4 B, C$ ), the data are inconsistent with separation of adjacent stereocilia.

At the same time, stereocilia must slide past one another. For a peak deflection of $0.5 \mu \mathrm{m}$ and frequency of $700 \mathrm{~Hz}$, the shear rate for the tallest stereocilia is $>200 \mathrm{~nm} / \mathrm{ms}$. This puts interesting constraints on possible mechanisms for sliding adhesion.
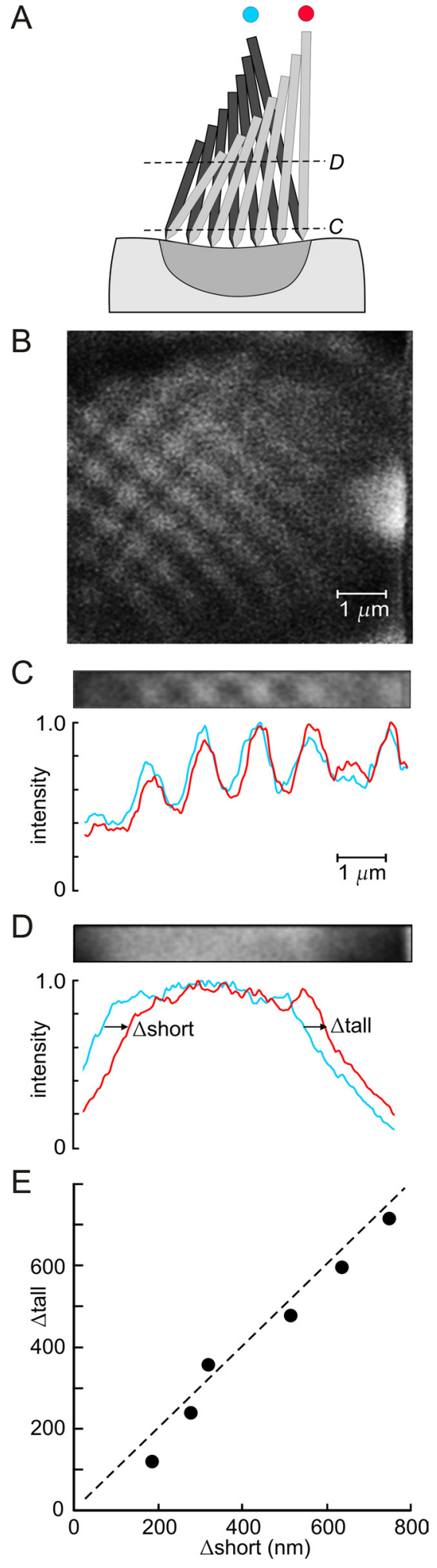

Figure 3. Two-photon imaging of a bundle in a whole mount of the saccule. $A$, Drawing of a hair bundle at rest (blue dot) and at peak excitatory displacement (red dot). The dashed lines indicate the focal levels for $\boldsymbol{C}$ and $\boldsymbol{D}$. $\boldsymbol{B}$, Image of a bundle near its base, looking from the top; membranes were labeled with FM1-43 in the bath. $\boldsymbol{C}$, Image and intensity profiles of a single column of stereocilia, for resting and deflected bundle positions (colors as in $\boldsymbol{A}$ ). Local peaks indicate individual stereocilia. D, Same column of stereocilia as in C at a higher focal level; individual stereocilia are not distinguishable. Motion is apparent from the shift of the intensity profile at the short and tall edges of the column. $\boldsymbol{E}$, Change in displacement of the tall versus the short edge of the column at different focal planes. The dashed line indicates equal displacement. 
A
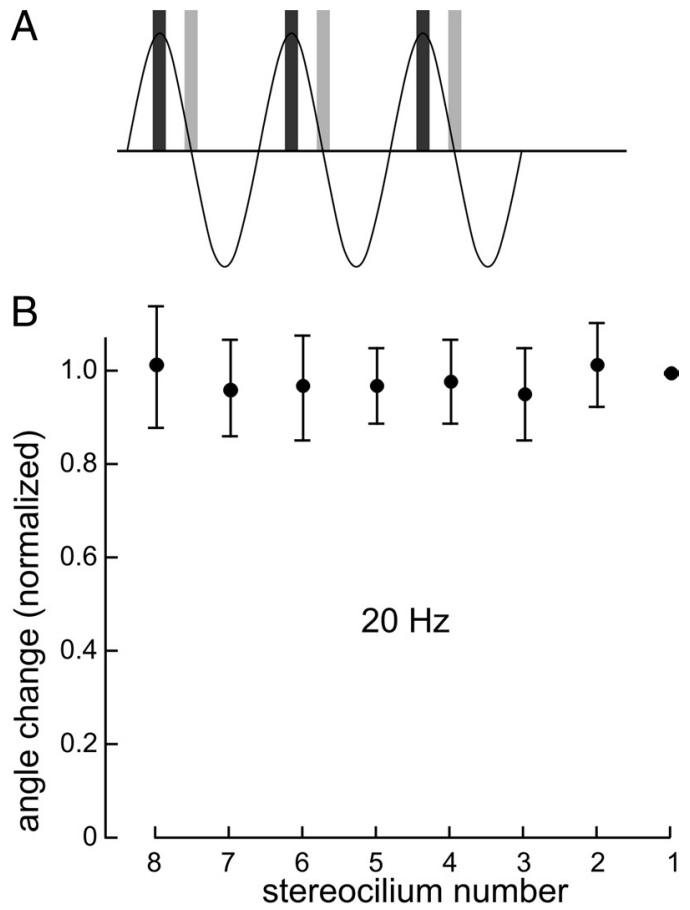

C

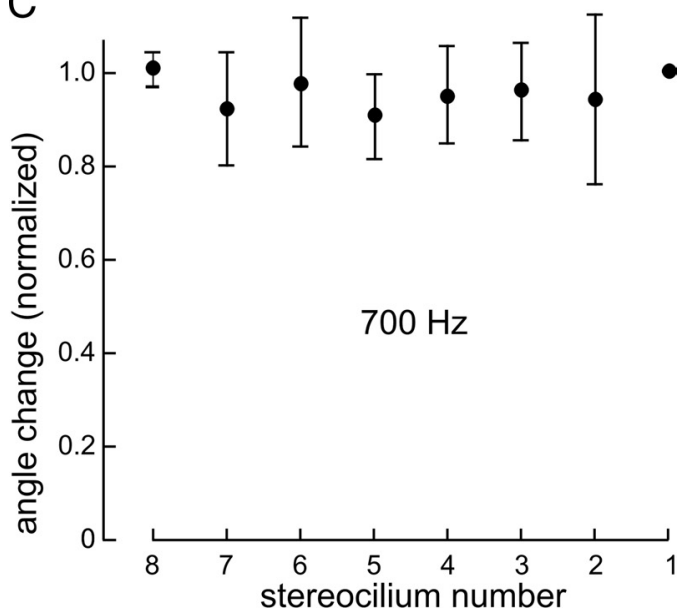

Figure 4. Hair bundle motion at high frequencies, for deflections of $\sim 0.5 \mu \mathrm{m}$ peak amplitude. $A$, Timing of the strobe flashes at two of eight time points (dark and light bars) along the stimulus cycle (sinusoid). $\boldsymbol{B}$, Angular displacement at $20 \mathrm{~Hz}$ measured from nine cells (mean \pm SD; same cells as in Fig. 1G). $\boldsymbol{C}$, Same as in $\boldsymbol{B}$ but at $700 \mathrm{~Hz}$. Data are normalized to the angular displacement of the tallest stereocilium for each experiment.

How do stereocilia remain together during bundle deflection? Cleaving links between stereocilia

We first confirmed that all types of links expected to be present in mature frog saccular hair cells were present in our preparations. Using transmission electron microscopy on preparations fixed without any enzyme treatment, we observed tip links (Fig. 5A), horizontal top connectors (Fig. $5 B$ ), and ankle links (Fig. 5C,D). There were also sparse links more distal than the taper region of stereocilia, which we assume are shaft connectors. If we used proteinase XXIV treatment to remove the otolithic membrane, as was done for all optical imaging experiments, the ankle links and shaft connectors were mostly absent (Fig. 5E) (Jacobs and Hudspeth, 1990; Bashtanov et al., 2004). When ankle links and shaft connectors were not altogether missing, we saw what appeared to be link remnants that were disorganized (Fig. 5F; compare with
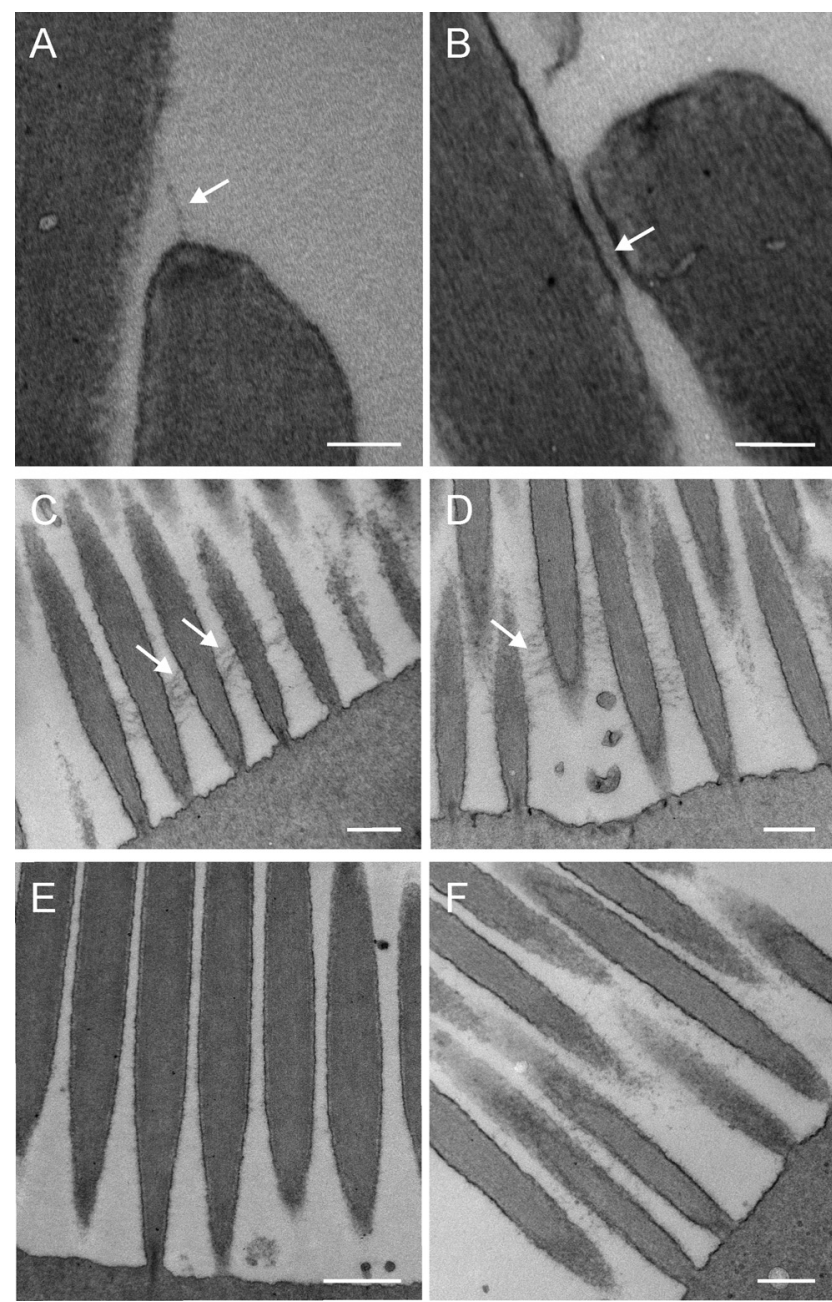

Figure 5. Links between stereocilia in our preparation of the bullfrog saccule; transmission electron microscopy. $\boldsymbol{A}$, Tip link. $\boldsymbol{B}$, Horizontal top connectors between adjacent stereocilia. $\boldsymbol{C}, \boldsymbol{D}$, Ankle links extending between adjacent stereocilia near their bases; no enzyme. $\boldsymbol{E}, \boldsymbol{F}$, In preparations treated with proteinase XXIV, ankle links were absent $(\boldsymbol{E})$ or appeared disorganized $(\boldsymbol{F})$. The arrows point to the corresponding links in each panel. Scale bars: $\boldsymbol{A}, \boldsymbol{B}, 100 \mathrm{~nm} ; \boldsymbol{C}-\boldsymbol{F}, 500 \mathrm{~nm}$.

Fig. $5 C, D)$ and in general did not span the entire distance between the stereocilia.

To investigate which links may be responsible for the propagation of the mechanical stimuli, we used biochemical treatments to selectively destroy certain links. In all imaging experiments, the ankle links and shaft connectors were compromised because we used proteinase XXIV to remove the otolithic membrane. As shown in Figures 1-4, bundles remained together in the absence of ankle links and shaft connectors.

Tip links in dissociated bullfrog hair cells break after a brief application of the $\mathrm{Ca}^{2+}$ chelator BAPTA (Assad et al., 1991). Using a micropipette placed $\sim 30 \mu \mathrm{m}$ away from a hair bundle, we applied BAPTA locally to break tip links. To test breakage, we then bath-applied FM1-43 and assessed labeling of intracellular organelles. When tip links are intact, dye enters the tips of stereocilia within seconds of dye application and passes into the cell body. After BAPTA treatment in these experiments, there was no dye entry into hair cell cytoplasm for at least $2 \mathrm{~min}$. We therefore concluded that the tip links were broken. Note that the tip links are thought to be composed of the same proteins as the kinocilial links that hold the kinocilium to the adjacent stereocilia (cadherin 23 and protocadherin 15) (Tsuprun et al., 2004; Kazmierc- 
zak et al., 2007); not surprisingly, the kinocilium often detached from the rest of the bundle after BAPTA treatment. In these cases, the stimulus probe was then attached with suction to the tallest stereocilia to test bundle motion.

When viewed with DIC imaging at both low and high frequencies, BAPTA-treated cells showed movement similar to nontreated cells. The measured angular deflections were similar for all stereocilia within the same column of the hair bundle, for all frequencies (Fig. 6A-C). We conclude that frog saccular stereocilia are not held together by either ankle links, shaft connectors, or tip links.

We did not find enzymatic conditions that cleave horizontal top connectors; since they are the only links remaining, they are candidates for mediating lateral adhesion. We tested this in several ways.

\section{Orthogonal stimuli}

To further test the nature of links that might hold stereocilia together, we chose hair bundles whose morphological axis was parallel to the light path, that is, pointing straight up or down. The stimulus probe was attached by suction to stereocilia of midheight at one edge of a bundle, and the bundle was moved perpendicular to the excitatory axis (Fig. $7 A, B$ ). As can be seen in Figure $7 C$, stereocilia stayed tightly together even for sideways deflections of $\sim 2 \mu \mathrm{m}$. We measured the movement of individual stereocilia of a row and fitted the movement with the touch model, setting stereocilia heights equal. The prediction fits the observed movement well, indicating that stereocilia remain in contact even for perpendicular stimuli. Thus, stereocilia are held together by a mechanism that is not oriented exclusively along a column, such as tip links, but that extends in all directions.

\section{Prestressed rootlets}

In similar experiments using interference microscopy, Kozlov et al. (2007) found that bullfrog saccular stereocilia remain touching when stimulated by brownian motion, even for frequencies up to $5 \mathrm{kHz}$. Maximum deflections in these experiments were less than $\sim 50 \mathrm{~nm}$. They noted that the cuticular plate is curved and that, if the stereocilia rootlets are oriented perpendicular to the cuticular plate, it would tend to push stereocilia tips together. Such curvature could hold the tips together simply by elastic forces in the pivots, in the absence of any additional adhesion mechanism.

To test whether this might hold for larger stimuli, we first asked whether the rootlets were indeed oriented perpendicular to the curvature, by measuring rootlets from electron micrographs (Fig. $8 A, B$ ). In fact, rootlets are not perpendicular but tend to be slightly bent as they insert into the cuticular plate, by an angle that might be predicted to push the tips toward the center.

We assessed the bend by measuring the angle of a rootlet relative to the angle of its stereocilium, for different stereocilia in a column (Fig. 8C). The angle is negative for tall stereocilia, whereas it is positive for short stereocilia. If the bend in the rootlets represents a resting stress, then the force produced by the bend would push tall stereocilia in the negative direction and would tend to push short stereocilia in the positive direction. Thus, not only does the curvature of the cuticular plate tend to orient stereocilia toward each other, but additional "prestress" in the bend of the rootlets may tend to push them together, at least to a point.

The maximum angle of prestressing is $\sim 7^{\circ}$ (Fig. $8 C$ ), so if this were the sole mechanism for maintaining bundle cohesion it should work for positive deflections up to $\sim 7^{\circ}(\sim 1 \mu \mathrm{m})$ but not beyond. We gave stimuli much larger than that to see whether
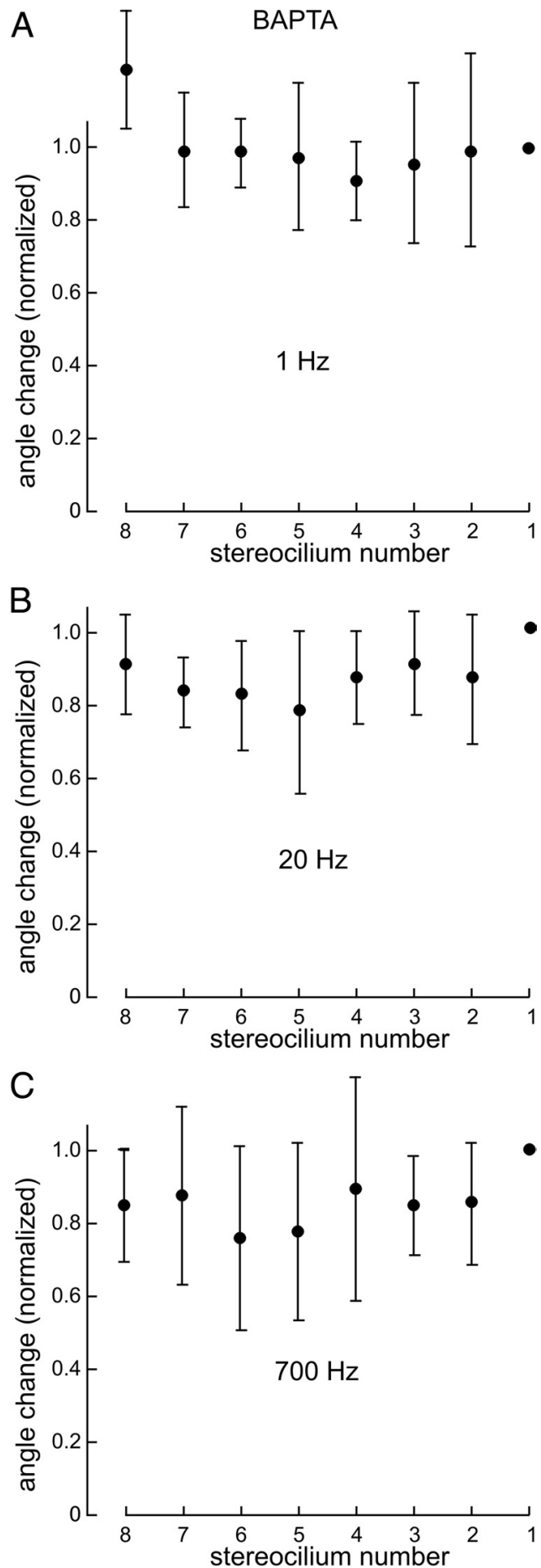

Figure 6. Hair bundle motion in BAPTA-treated cells. $\boldsymbol{A}$-C, Angular displacement calculated from six cells at 1, 20, and $700 \mathrm{~Hz}$, respectively. Data are normalized to the displacement of the tallest stereocilium (no. 1) for each experiment. Stimulus displacement $<500 \mathrm{~nm}$. Error bars indicate SD.

stereocilia separated. Figure $9, A$ and $B$, shows a hair bundle deflected in the positive direction by $31^{\circ}(\sim 4.5 \mu \mathrm{m})$ from rest. Although the stimulus probe pulled only on the bulb of the kinocilium, all the stereocilia remained in contact. The movement of individual stereocilia was measured from images (Fig. 9C) and fitted with predictions of the geometrical model in which stereocilia are presumed to remain touching (Fig. 9D). Even for large deflections $\gg 7^{\circ}$, the touch model provides a good fit. 

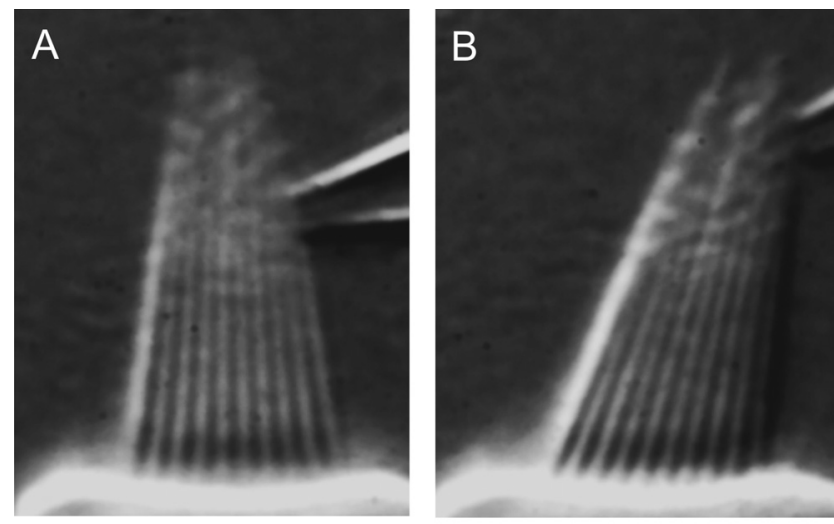

C

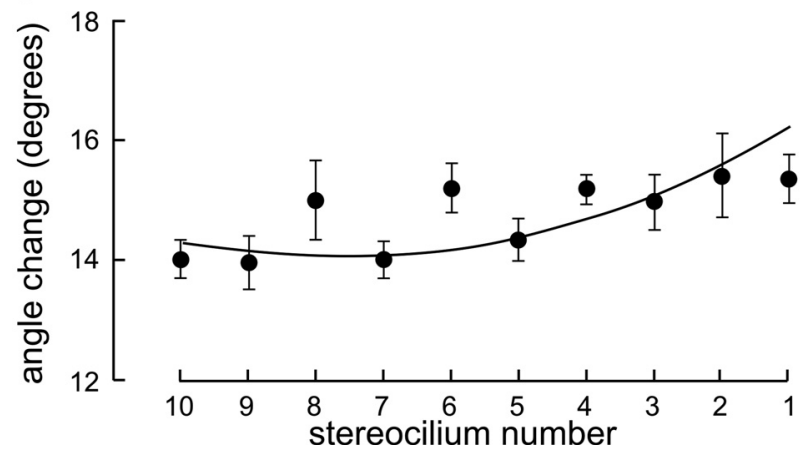

Figure 7. Orthogonal hair bundle stimulation. $\boldsymbol{A}, \mathrm{DIC}$ image of a hair bundle viewed perpendicular to its excitatory axis. A stimulus probe was attached to the right edge of the bundle. $B$, Static stimulus of $\sim 2 \mu \mathrm{m}$, perpendicular to the excitatory axis. C, Angular displacement of each stereocilium in an image plane near the shortest row (SD is calculated from all the extractions along the length of each stereocilium). The line indicates the fit of the touch model.

Perhaps the angles of the rootlets are not indicative of the extent of prestressing, and stereocilia are really pressed together more tightly than rootlet angles suggest. Then they might remain in contact even for large deflections. We tested this by combing the hair bundle with a fine glass pipette (Fig. 10 A). We first observed cohesion of stereocilia for large deflections (Fig. $10 \mathrm{~B}$ ), and then returned the bundle to the rest position. The pipette was inserted between stereocilia near the ankle region, from the side (Fig. 10C), and then lifted up toward the tips (Fig. 10D). We reasoned that combing in this way would disrupt any lateral links that might hold the bundle together but would not affect prestressing of rootlets. When the bundle was again given a large positive deflection, only the taller half of the stereocilia followed the stimulus probe (Fig. $10 \mathrm{E}$ ). The short half remained at the rest position (compare with Fig. 10C), indicating that they were not intrinsically stressed toward the positive direction. Thus, prestressing of stereocilia rootlets may contribute to maintaining stereocilia contact, but it seems unlikely to be the mechanism that holds them together for large deflections.

We then asked whether the links that are disrupted by combing can reattach. We first tried pushing the taller half of the bundle back toward the short half after combing. In some cases, the bundle recombined, although the apparent adhesion strength was much reduced. To further explore the reversibility of attachments, we mechanically removed individual stereocilia by blotting them on a cover glass, as previously described (Shepherd et al., 1990). Individual stereocilia were then held by a glass probe with suction and brought into contact with another stereocilium. The two stereocilia often appeared to stick to each other, espe-
A

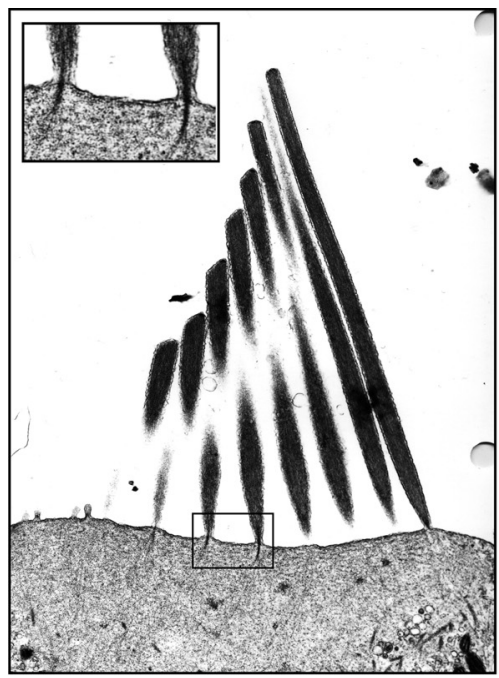

B

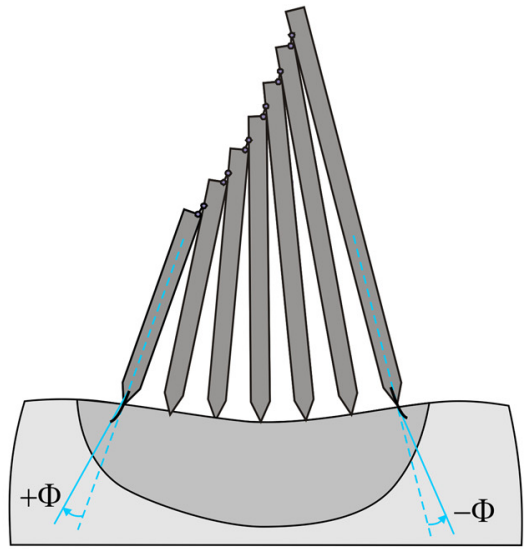

C

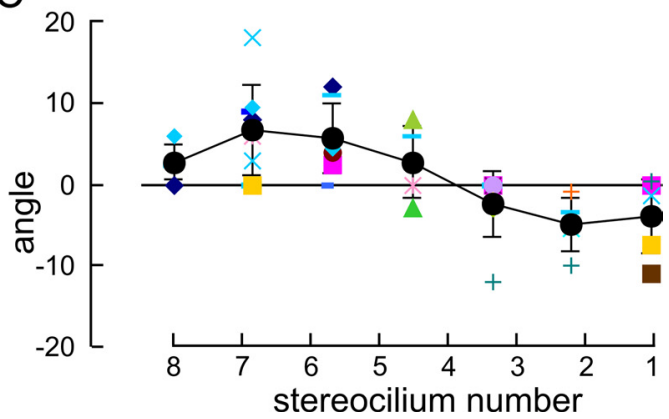

Figure 8. Angles of stereocilia rootlets. $A$, Transmission electron micrograph of a hair bundle where the rootlets of two stereocilia are visible (magnified in the inset). $\boldsymbol{B}$, Method for calculating the rootlet angles. The center line of a stereocilium (dashed) was extended into the cell body, and the angle of the rootlet (solid line) was measured from it. C, Measurements of rootlet angles from 20 cells. The black symbols and lines indicate the mean and SD. Rootlets angled outward by up to $\sim 7^{\circ}$, which may push stereocilia tips together.

cially near their tips (supplemental Fig. S1, available at www. jneurosci.org as supplemental material), suggesting the presence of an intrinsic adhesive mechanism.

Thus, it seems likely that some sort of lateral link maintains stereocilia cohesion. Bundles stay together after enzymatic digestion of the ankle links and shaft connectors and after BAPTA cleavage of tip links, when the only remaining links are the horizontal top connectors. Horizontal top connectors extend among stereocilia in all directions [our unpublished observation in frog and in chicken (Goodyear and Richardson, 1992; Tsuprun and 

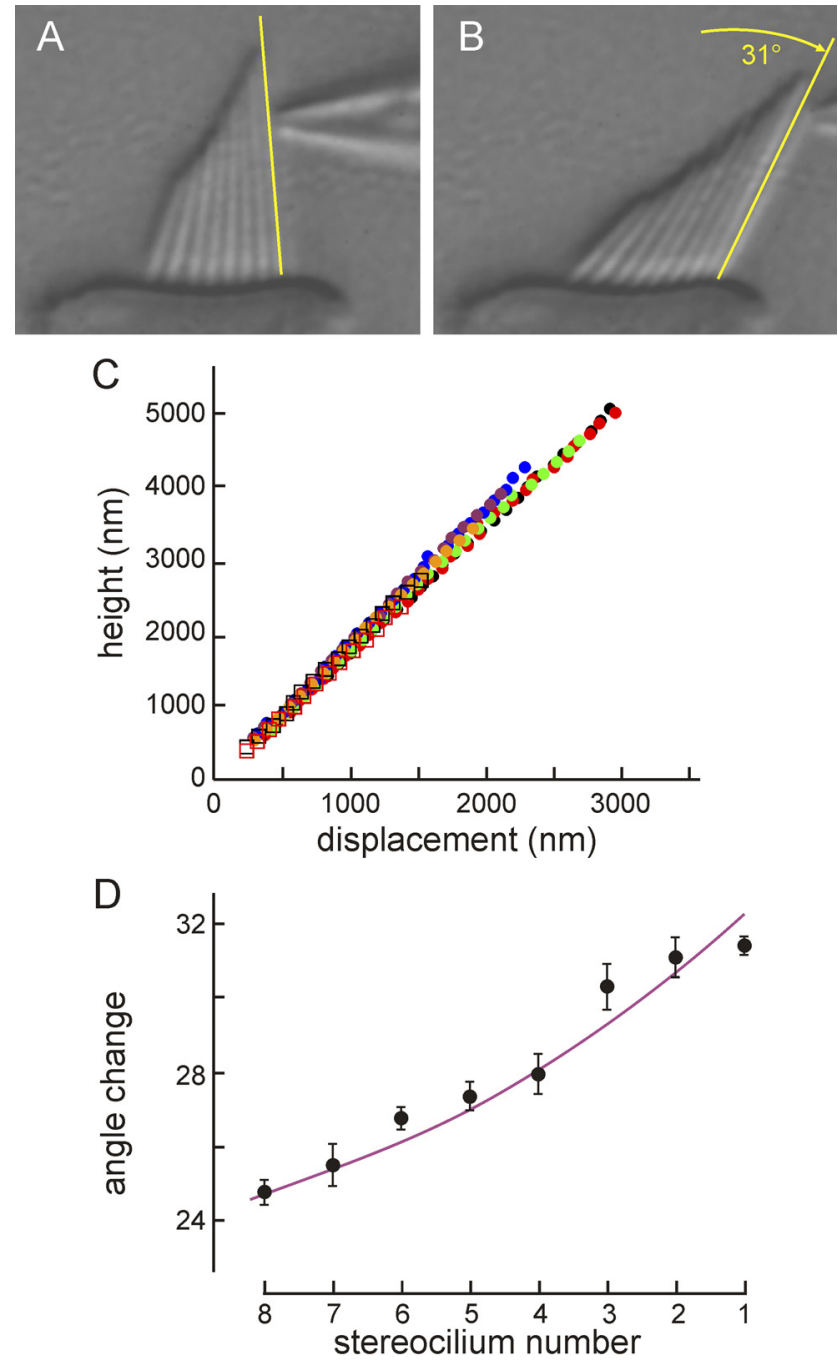

Figure 9. Hair bundle motion in response to a large static stimulus. $A, B$, DIC images of a hair bundle deflected by $\sim 4.5 \mu \mathrm{m}$ or $31^{\circ}$ in the excitatory direction. $C$, Peak displacements of individual stereocilia along the same column for several locations along their heights. D, Angular displacement of each stereocilium; the line indicates predictions of the touch model. Mean and SD calculated from all the extractions along the length of each stereocilium.

Santi, 1998)] so they could also maintain cohesion for large sideways deflections (Fig. 7).

Bundle cohesion in the absence of horizontal top connectors

In the chicken utricle, extrastriolar hair cell bundles have an extensive array of shaft connectors but lack morphologically distinct horizontal top connectors (Goodyear and Richardson, 1992). We tested bundle cohesion in these hair cells from E16E20 chick embryos, with and without treatments reported to remove all links (Bashtanov et al., 2004). In control cells, most of the bundles moved as a unit, with shortest and tallest stereocilia moving through similar angles in response to large $(>5 \mu \mathrm{m})$ deflections (Fig. 11 $A, B$ ). However, even control bundles seemed more fragile than frog saccular hair cells, as $\sim 25 \%$ of the cells tested showed some minor splay during large deflection. When the epithelia were instead treated with proteinase XXIV for 22 min followed by BAPTA, which cuts ankle links, shaft connectors, and tip links (Bashtanov et al., 2004), all of the cells splayed during deflections (Fig. 11C,D). Bundle cohesion appears compromised in hair bundles that lack horizontal top connectors, especially when all other links are removed.
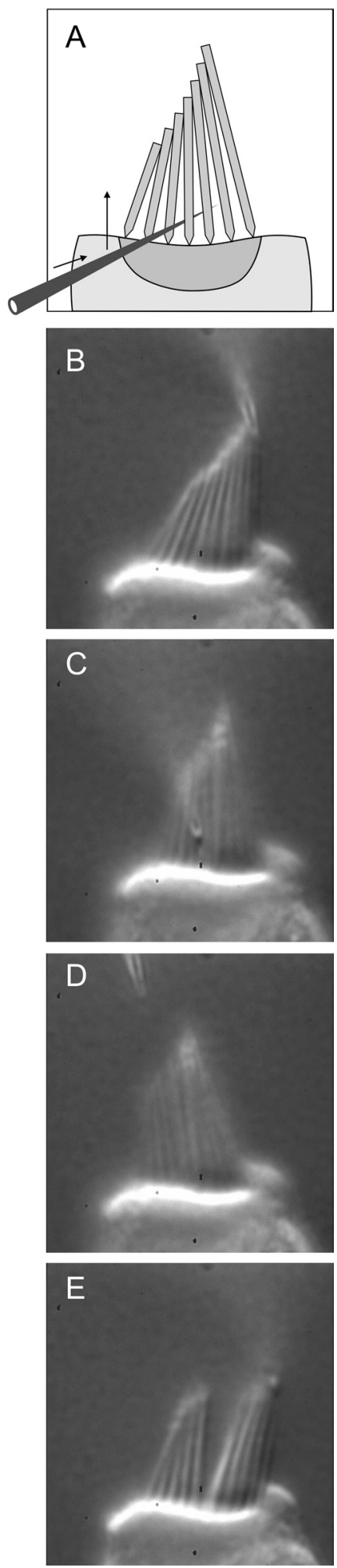

Figure 10. Combing away links between adjacent stereocilia. $A$, The tip of a fine glass probe ( $\sim 50 \mathrm{~nm}$ tip diameter) was inserted through the bundle between stereocilia at their bases and then lifted toward their tips (arrows). B, The combing probe was first used to displace the hair bundle and observe bundle cohesion. $C$, The probe tip was inserted between stereocilia near the ankle region; the tip is not visible here as it was bent to run perpendicular to the image plane. $\boldsymbol{D}$, The probe was lifted toward the tip and out of the bundle. $\boldsymbol{E}$, The probe was used again to deflect the bundle, revealing lack of cohesion at the combing site. 

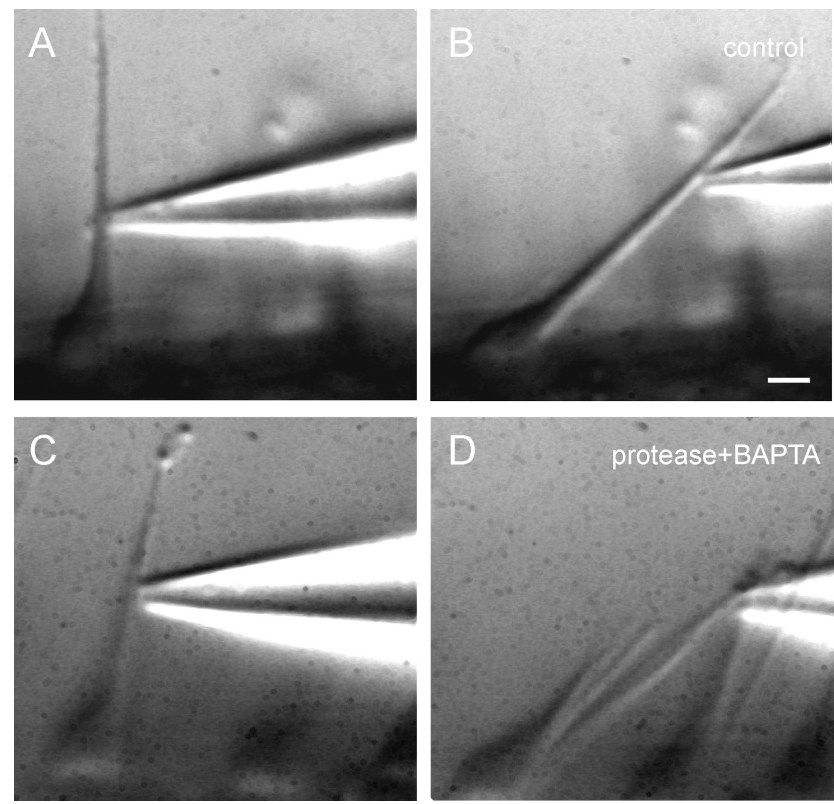

Figure 11. Chick utricle hair cells. Hair cells from the extrastriolar region of E16-E20 chick utricles were viewed from the side, by folding the intact epithelium and selecting hair cell bundles extending parallel to the chamber bottom. $A$, A control hair bundle, undeflected. $\boldsymbol{B}$, The same bundle deflected by $>8 \mu \mathrm{m}$ by pulling on the tallest stereocilia with a suction pipette. № splay was evident. $C$, A bundle treated with protease and BAPTA. D, The same bundle deflected. Stereocilia tended to pull apart under these conditions. Scale bar, $2 \mu \mathrm{m}$.

\section{Charge and ionic interactions}

Stereocilia have an extensive negatively charged glycocalyx (Santi and Anderson, 1987). Theoretical work predicts that, if the coat charge is equally distributed within the glycocalyx or is concentrated near the outer surface, then electrostatic repulsion is able to maintain spatial separation between stereocilia (Dolgobrodov et al., 2000a,b). A separate mechanism would then be required to prevent additional separation and to provide an adhesive force (Flock et al., 1977; Howard et al., 1988; Hudspeth, 1992).

We explored the effects of charge interactions on bundle adhesion by changing the ionic strength, multivalent ion concentration, and $\mathrm{pH}$ of the bathing solution. In these experiments, the epithelia were first treated with proteinase XXIV and then with 5 mM BAPTA to remove all links except the horizontal top connectors, unless mentioned otherwise.

If the negatively charged glycocalyx produces repulsion when stereocilia membranes come in close proximity, then multivalent cations might both negate that charge and form electrostatic bridges between adjacent stereocilia. Indeed, polycations such as ruthenium red cause stereocilia to fuse near their tops under such conditions (Neugebauer and Thurm, 1987). Consistent with this, $100 \mathrm{mM} \mathrm{La}^{3+}$ added to the medium caused the stereocilia to pull together more closely, and they appeared to have fused together near their tips (Fig. 12A). When we applied displacement stimuli to such bundles, adjacent stereocilia did not slide against each other and deflection of the bundle moved the entire cell (data not shown). $\mathrm{La}^{3+}$ thus appears to lock together adjacent stereocilia, preventing sliding adhesion.

Neugebauer and Thurm (1987) found that low ionic strength solutions with only $0.2 \mathrm{~mm} \mathrm{Mg}{ }^{2+}$ produced a separation of hair bundles but did not eliminate horizontal top connectors. If the horizontal top connectors are proteinaceous, it might be that the low concentration of $\mathrm{Mg}^{2+}$ was sufficient to bridge acidic residues in extracellular protein domains on adjacent stereocilia,
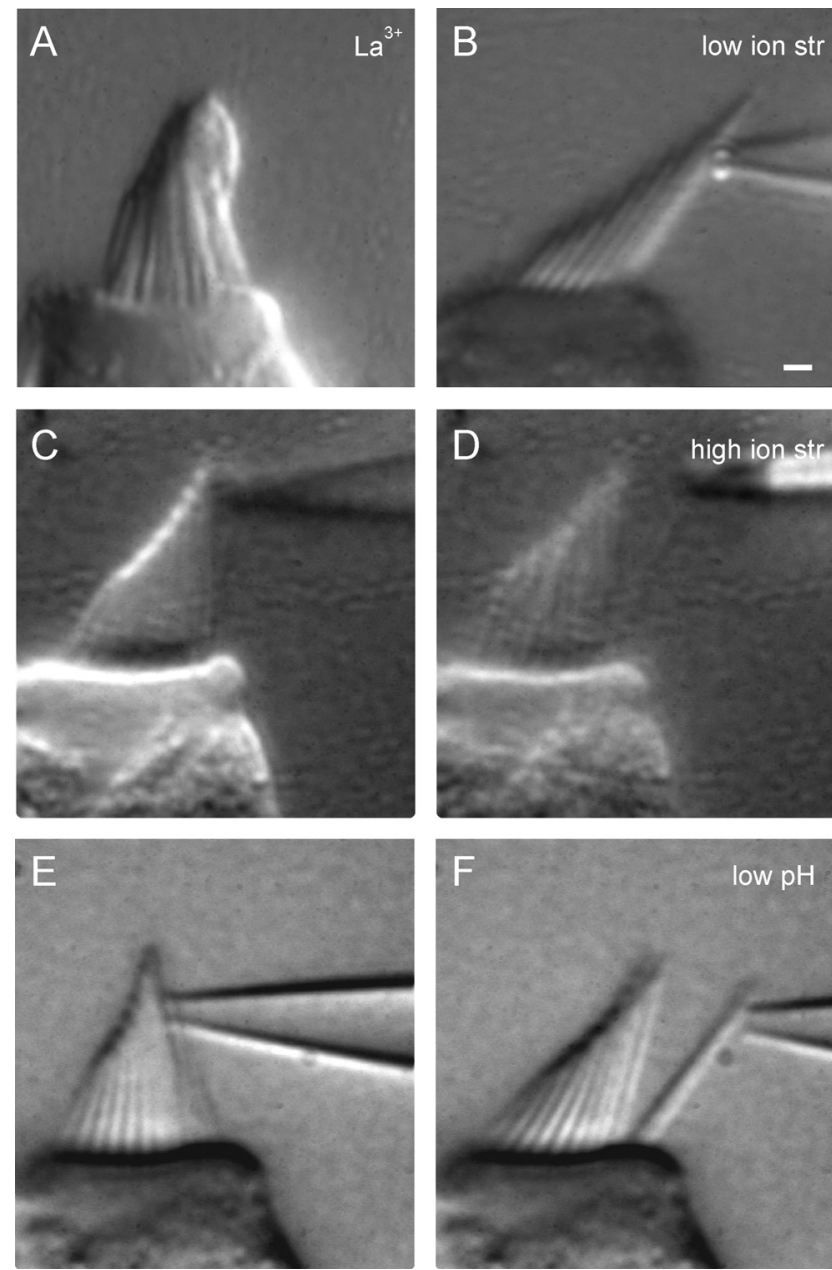

Figure 12. Ionic dependence of bundle cohesion in frog saccular hair cells. $\boldsymbol{A}$, In the presence of $100 \mathrm{~mm} \mathrm{La}^{3+}$. Stereocilia appear pinched together and the bundle is twisted. $\boldsymbol{B}$, Another bundle in a medium of low ionic strength and no divalent cations. Bundle cohesion is normal. $\boldsymbol{C}$, $D, A$ bundle during the application of $5 \mathrm{~m} \mathrm{NaCl}$ medium. Stereocilia tended to splay, and the bundle could be pulled apart by the stimulus probe. $\boldsymbol{E}, \boldsymbol{F}, \mathrm{A}$ bundle in medium of normal ionic strength but $\mathrm{pH}$ 3. The bundle was pulled apart by the stimulus probe. Scale bar, $1 \mu \mathrm{m}$.

similar to the $\mathrm{Ca}^{2+}$ stabilization of the extracellular domains in cadherin 23 (Sotomayor et al., 2010). We tested the effect of low ionic strength by replacing all ions in the solution with D-sorbitol to maintain osmolarity (except the HEPES and $\mathrm{NaOH}$ needed to adjust $\mathrm{pH} ; \sim 5 \mathrm{~mm}$ ). The bundle remained cohesive even for large deflections (Fig. 12 B), ruling out an ionic bridge in adhesion but not inconsistent with a role for the horizontal top connectors.

Perhaps an extracellular protein domain has both positive and negative charges, separated by several nanometers, that attract their opposites on strands from an adjacent stereocilium. Then it may be possible to disrupt these interactions with high ionic strength. We explored this idea by increasing the concentration of $\mathrm{NaCl}$ in the medium from $120 \mathrm{~mm}$ to $5 \mathrm{M}$. The bundle remained cohesive for hypertonic solutions of $<3 \mathrm{M} \mathrm{NaCl}$, but stereocilia tended to splay for larger concentrations of $\mathrm{NaCl}$ (Fig. $12 C, D$ ) (for the bundle shown in these panels, there was no BAPTA application). High osmolarity but normal ionic strength did not produce splay. It is possible that the splay was not caused by a specific effect of high ionic strength but from a nonspecific effect of the rapid deterioration of the cells that occurred under these conditions. Consequently, we varied the $\mathrm{pH}$ while maintaining normal ionic strength. Bundle cohesion remained normal 
up to $\mathrm{pH}$ 10; beyond that, the cells rapidly died. We then lowered the $\mathrm{pH}$ of the medium, by either exchanging LCR with the low $\mathrm{pH}$ medium or by using a pipette to puff low-pH solutions onto bundles, and looked for rapid effects that might be related to protonation of acidic residues. Conspicuous splay became evident as the $\mathrm{pH}$ approached 3; stereocilia separated but remained straight and pivoted at their bases (Fig. 12 E,F). Additional exposure to such low $\mathrm{pH}$ caused the stereocilia to lose their stiffness and to adopt a significantly curved shape, but the splay was evident within seconds and before the stiffness was lost.

\section{Discussion}

\section{Stereocilia move as rigid rods and pivot at their bases}

Stereocilium displacement is proportional to height for small and large displacements, and for low and high frequencies. Stereocilia thus move as rigid rods that pivot at their insertion points, consistent with previous observations that hair bundle stiffness decreases as the inverse square of stimulus height (Crawford and Fettiplace, 1985; Howard and Ashmore, 1986).

\section{The hair bundle moves as a unit}

High-resolution video images suggest that stereocilia remain close together as they move. Measurements (Figs. 1G, 2A, 7C, $9 D)$ confirmed this: stereocilia displacements match the predictions of a geometrical model that assumes adjacent stereocilia remain touching at all times. For small stimuli $(<0.5 \mu \mathrm{m})$, all stereocilia in the same column of the bundle moved with similar angular displacements, whereas for very large positive stimuli (up to $4.5 \mu \mathrm{m}$ ) short stereocilia moved less than tall stereocilia-as is also predicted by the touch model. The same behavior was observed at low and high frequencies, with no phase lag between tallest and shortest stereocilia. Based on the goodness of fit of geometric models, we estimate that pairs of stereocilia in a column do not increase their separation by more than $\sim 5 \mathrm{~nm}$ for a positive deflection at the tip of the bundle of $<300 \mathrm{~nm}$. The same mechanism appears to hold for large stimuli, putting considerable constraints on the mechanism of bundle cohesion.

\section{Horizontal top connectors may mediate coherent motion}

What holds stereocilia together? These experiments rule out a number of mechanisms. Tip links do not hold stereocilia together, because BAPTA-treated bundles move the same as untreated bundles (Fig. 6) and because stimuli perpendicular to tip links also move stereocilia as a unit (Fig. 7). Similarly, Kozlov et al. (2007) found that models with a tip link stiffness even 50 times larger than physiological estimates could not produce the coherence they observed. Ankle links and shaft connectors do not hold stereocilia together, because the enzyme used for removing the otolithic membrane also digests these links (Fig. 5) (Bashtanov et al., 2004).

Viscous coupling between adjacent stereocilia might entrain the bundle to move as a whole. Viscous forces are more important than inertial forces at auditory frequencies (Weiss and Leong, 1985; Freeman and Weiss, 1988). Are they also more important than elastic forces? Zetes and Steele (1997) found that they become more important for frequencies above $\sim 1 \mathrm{kHz}$, but the effect was to produce additional splay as a consequence of drag rather than to entrain stereocilia. At lower frequencies, the stereocilia pivot stiffness and the inter-stereocilia coupling stiffness dominated the bundle motion. Thus, viscous forces cannot hold stereocilia together at low frequency.

Perhaps the curvature of the cuticular plate in frog saccule tilts stereocilia together and holds them in contact (Kozlov et al., 2007). The dimensions of this curvature-and an additional
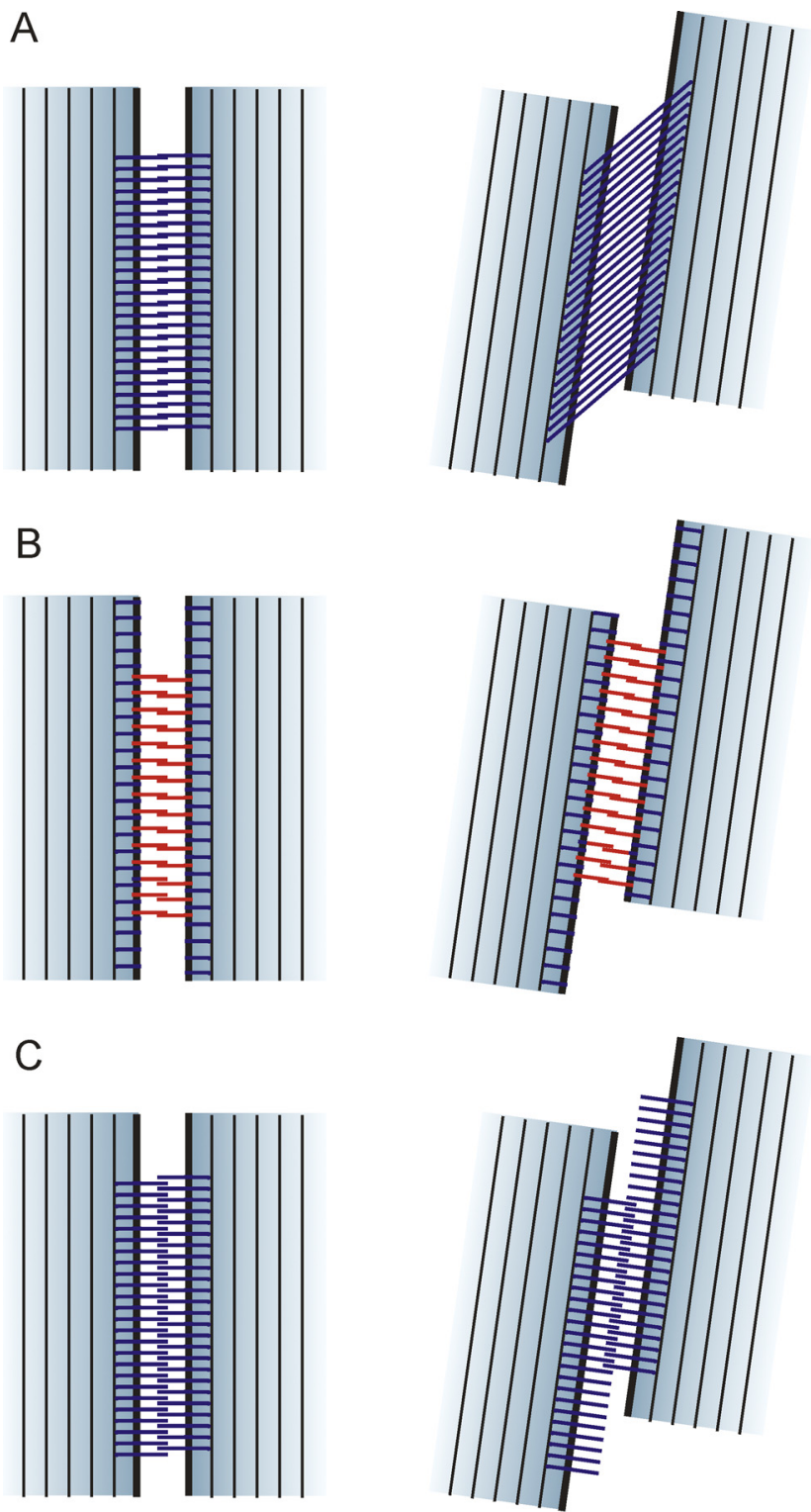

D

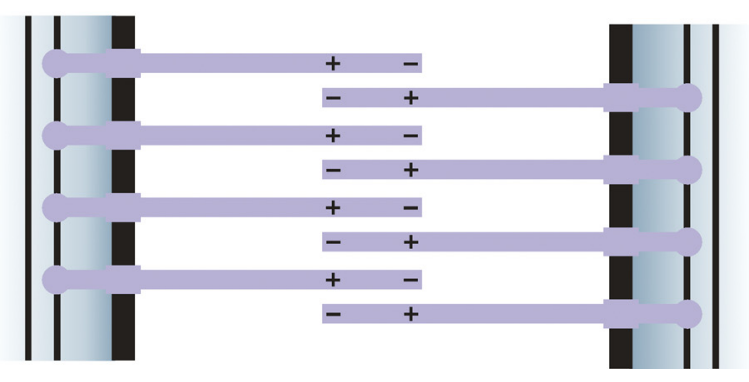

Figure 13. Models for sliding adhesion. $\boldsymbol{A}$, Fixed extensible links. Links might be anchored to the actin cores but must be able to stretch many times their length. $\boldsymbol{B}$, Inextensible links anchored to the lipid (red) might be able to slip laterally; a separate set of fixed, actin-bound links (blue) would prevent lipid detachment. $\boldsymbol{C}$, Inextensible links might be anchored to the actin, but their extracellular domains might have a loose electrostatic attraction that allows slipping. D, A possible arrangement of charge that could provide electrostatic attraction without dependence on bridging ions.

bend of the rootlets-suggest that rootlet bending forces could hold stereocilia together, but only to $\sim 7^{\circ}$ deflection. Yet stereocilia remain touching with much larger deflections (Fig. 9), indicating the presence of an additional, probably more universal 
mechanism. Moreover, combing the bundle with a micropipette allowed the taller part of the bundle to move with an excitatory stimulus while the shorter part remained upright and stationary, indicating that the natural bias of the shorter stereocilia is not strongly toward the excitatory direction (Fig. 10).

Horizontal top connectors have been suggested to participate in bundle cohesion (Pickles et al., 1984; Howard et al., 1988; Jacobs and Hudspeth, 1990; Geisler, 1993; Pickles, 1993). Here, we find that frog bundles stay together when horizontal top connectors are the only known link remaining. Conversely, extrastriolar hair cells from the chicken utricle, which lack horizontal top connectors (Goodyear and Richardson, 1992), can splay after treatments that remove all other links (Bashtanov et al., 2004). If a specific set of links holds the bundle together, it is most likely these.

\section{How might horizontal connectors mediate adhesion?}

The geometry of stereocilia creates special constraints for any sort of lateral link that maintains bundle cohesion (Howard and Ashmore, 1986). Our measurements indicate that, with large displacements, stereocilia must shear by $500 \mathrm{~nm}$ or more, while not increasing their separation by $>70 \mathrm{~nm}$. This shear must also occur at high speeds $(>250 \mathrm{~nm} / \mathrm{ms}$ for a $500 \mathrm{~nm}, 700 \mathrm{~Hz}$ stimulus). Horizontal top connectors might mediate adhesion by stretching (Fig. 13A). However, it is hard to imagine how they could stretch hundreds of nanometers when the interstereociliary spacing is $\sim 20 \mathrm{~nm}$ at the tips of frog stereocilia. In addition, such stretching would contribute a large, nonlinear stiffness to the bundle when the shear is much more than the separation (within the physiological range of deflections), which has not been observed in frog.

More likely is a kind of sliding adhesion that prevents separation of stereocilia membranes but allows them to slide relative to one another in the plane of the membrane (Fig. $13 \mathrm{~B}, \mathrm{C}$ ). Horizontal top connectors have been described in frog as amorphous mats (Jacobs and Hudspeth, 1990; Nagel et al., 1991), in chicken as short bars in a hexagonal array (Goodyear and Richardson, 1992), and in mammalian outer hair cells as a zipper-like lattice (van Benthem et al., 1993; Tsuprun and Santi, 1998, 2002; Tsuprun et al., 2003; Goodyear et al., 2005). All are generally consistent with filaments extending from neighboring stereocilia and overlapping in the middle. It might be that filaments bind tightly at the overlap; if so, sliding must occur by lateral mobility in the membrane, with an immobile intracellular protein holding the membrane to the actin core (Fig. 13B). Alternatively, sliding adhesion might be mediated by a more fluid electrostatic interaction between stereocilia (Flock et al., 1977; Howard et al., 1988; Hudspeth, 1992), specifically between extracellular domains of the horizontal top connectors (Fig. 13C). Our experiments showing opposite effects of $\mathrm{La}^{3+}$ and low $\mathrm{pH}$ suggest that stereocilia separation and bundle cohesion rely on a delicate balance between membrane repulsion caused by a negatively charged glycocalyx, and an electrostatic linkage that can be protonated (Fig. 13D).

Stereocilin, a protein defective in the human recessive deafness DFNB16, is associated with the horizontal top connectors in mouse outer hair cells (Verpy et al., 2001, 2008). It is an acidic, secreted, extracellular protein that may be linked to the stereocilia membrane by a GPI (glycosylphosphatidylinositol) anchor, and it is thought to have a superhelical structure that may extend 20-25 nm from each membrane (Sathyanarayana et al., 2009). The size is consistent with horizontal top connectors in mammalian cochlea of 40-50 nm (Goodyear et al., 2005), but perhaps not with the shorter $(\sim 20 \mathrm{~nm})$ connectors observed in frog and chick (Fig. 5B) (Goodyear and Richardson, 1992). An evaluation of bundle mechanics in stereocilin knock-out mice might shed light on its involvement in bundle cohesion.

\section{Sliding adhesion confers parallel gating to transduction channels}

The geometrical model calculates the stretch of gating springs for stereocilia of different heights. In the touch model, stretch is nearly equal for all stereocilia of a bundle, varying by $<1.7 \%$ for deflections up to $300 \mathrm{~nm}$ (Corey et al., 1989; Jacobs and Hudspeth, 1990; Geisler, 1993; Pickles, 1993). The change in force on transduction channels is proportional to stretch if all gating springs have similar stiffness. Thus, all channels get essentially the same stimulus.

In addition, if elements other than tip links hold stereocilia tightly together, then transduction channels are mechanically in parallel (Karavitaki and Corey, 2005; Kozlov et al., 2007). The opening or closing of channels does not affect the separation of stereocilia, and-for a displacement stimulus - the channels open and close independently. With a force stimulus, however, the parallel arrangement of channels can lead to positive cooperativity. If one channel opens, reducing tension in that gating spring, then the bundle can move a bit further, increasing tension in all other gating springs and making other channels more likely to open. Similarly, one channel closing would tend to make other channels close.

$\mathrm{Ca}^{2+}$ imaging has suggested that transduction channels, estimated at two per tip link, are only at the lower ends of tip links (Beurg et al., 2009). This complicates the biophysical model, as those two channels may interact mechanically depending on where the gating springs are located. If each channel has its own gating spring, however, then they also operate independently and all channels of a hair cell are mechanically in parallel (Corey, 2009).

Mechanisms associated with channel gating, including $\mathrm{Ca}^{2+}$. induced channel closure, the negative compliance of channel gating, and the $\mathrm{Ca}^{2+}$-dependent movement of myosins, may produce a positive feedback that amplifies the movement of a hair bundle (Howard et al., 1988; Choe et al., 1998; Martin et al., 2000; Cheung and Corey, 2006; Tinevez et al., 2007). Amplification by positive feedback can be degraded by mechanical noise such as brownian motion, but parallel coupling between adjacent hair bundles through an otolithic or tectorial membrane can reduce noise to restore amplification and tuning (Dierkes et al., 2008). The parallel coupling of transduction elements within a hair bundle by tight coupling between stereocilia has the same effect (Nam and Fettiplace, 2008). The unusual property of sliding adhesion between stereocilia membranes may have evolved to enhance an amplification mechanism associated with the mechanics of transduction.

\section{References}

Assad JA, Corey DP (1992) An active motor model for adaptation by vertebrate hair cells. J Neurosci 12:3291-3309.

Assad JA, Shepherd GM, Corey DP (1991) Tip-link integrity and mechanical transduction in vertebrate hair cells. Neuron 7:985-994.

Bashtanov ME, Goodyear RJ, Richardson GP, Russell IJ (2004) The mechanical properties of chick (Gallus domesticus) sensory hair bundles: relative contributions of structures sensitive to calcium chelation and subtilisin treatment. J Physiol 559:287-299.

Beurg M, Fettiplace R, Nam JH, Ricci AJ (2009) Localization of inner hair cell mechanotransducer channels using high-speed calcium imaging. Nat Neurosci 12:553-558.

Carter AG, Sabatini BL (2004) State-dependent calcium signaling in dendritic spines of striatal medium spiny neurons. Neuron 44:483-493.

Cheung EL, Corey DP (2006) $\mathrm{Ca}^{2+}$ changes the force sensitivity of the haircell transduction channel. Biophys J 90:124-139. 
Choe Y, Magnasco MO, Hudspeth AJ (1998) A model for amplification of hair-bundle motion by cyclical binding of $\mathrm{Ca}^{2+}$ to mechanoelectricaltransduction channels. Proc Natl Acad Sci U S A 95:15321-15326.

Corey DP (2009) Cell biology of mechanotransduction in inner-ear hair cells. F1000 Biol Rep 1:58.

Corey DP, Hudspeth AJ (1980) Mechanical stimulation and micromanipulation with piezoelectric bimorph elements. J Neurosci Methods 3:183-202.

Corey DP, Hacohen N, Huang PL, Assad JA (1989) Hair cell stereocilia bend at their bases and touch at their tips. Soc Neurosci Abstr 15:208.

Crawford AC, Fettiplace R (1985) The mechanical properties of ciliary bundles of turtle cochlear hair cells. J Physiol 364:359-379.

Crawford AC, Evans MG, Fettiplace R (1989) Activation and adaptation of transducer currents in turtle hair cells. J Physiol 419:405-434.

Dierkes K, Lindner B, Jülicher F (2008) Enhancement of sensitivity gain and frequency tuning by coupling of active hair bundles. Proc Natl Acad Sci U S A 105:18669-18674.

Dolgobrodov SG, Lukashkin AN, Russell IJ (2000a) Electrostatic interaction between stereocilia: I. Its role in supporting the structure of the hair bundle. Hear Res 150:83-93.

Dolgobrodov SG, Lukashkin AN, Russell IJ (2000b) Electrostatic interaction between stereocilia: II. Influence on the mechanical properties of the hair bundle. Hear Res 150:94-103.

Duncan RK, Hernandez HN, Saunders JC (1995) Relative stereocilia motion of chick cochlear hair cells during high-frequency water-jet stimulation. Aud Neurosci 1:321-329.

Duncan RK, Eisen MD, Saunders JC (1999) Distal separation of chick cochlear hair cell stereocilia: analysis of contact-constraint models. Hear Res 127:22-30.

Flock A, Flock B, Murray E (1977) Studies on the sensory hairs of receptor cells in the inner ear. Acta Otolaryngol 83:85-91.

Freeman DM, Weiss TF (1988) The role of fluid inertia in mechanical stimulation of hair cells. Hear Res 35:201-207.

Gale JE, Marcotti W, Kennedy HJ, Kros CJ, Richardson GP (2001) FM1-43 dye behaves as a permeant blocker of the hair-cell mechanotransducer channel. J Neurosci 21:7013-7025.

Geisler CD (1993) A model of stereociliary tip-link stretches. Hear Res 65:79-82.

Goodyear R, Richardson G (1992) Distribution of the $275 \mathrm{kD}$ hair cell antigen and cell surface specialisations on auditory and vestibular hair bundles in the chicken inner ear. J Comp Neurol 325:243-256.

Goodyear RJ, Marcotti W, Kros CJ, Richardson GP (2005) Development and properties of stereociliary link types in hair cells of the mouse cochlea. J Comp Neurol 485:75-85.

Howard J, Ashmore JF (1986) Stiffness of sensory hair bundles in the sacculus of the frog. Hear Res 23:93-104.

Howard J, Roberts WM, Hudspeth AJ (1988) Mechanoelectrical transduction by hair cells. Annu Rev Biophys Biophys Chem 17:99-124.

Hudspeth AJ (1983) The hair cells of the inner ear. They are exquisitely sensitive transducers that in human beings mediate the senses of hearing and balance. A tiny force applied to the top of the cell produces an electrical signal at the bottom. Sci Am 248:54-64.

Hudspeth AJ (1992) Hair-bundle mechanics and a model for mechanoelectrical transduction by hair cells. Soc Gen Physiol Ser 47:357-370.

Hudspeth AJ, Corey DP (1978) Controlled bending of high-resistance glass microelectrodes. Am J Physiol 234:C56-C57.

Jacobs RA, Hudspeth AJ (1990) Ultrastructural correlates of mechanoelectrical transduction in hair cells of the bullfrog's internal ear. Cold Spring Harb Symp Quant Biol 55:547-561.

Karavitaki KD, Corey DP (2005) Hair bundle mechanics at high frequencies: a test of series or parallel transduction. In: Auditory mechanisms: processes and models (Nuttall AL, Ren T, Gillespie P, Grosh K, de Boer E, eds), pp 286-292. Hackensack, NJ: World Scientific.

Karavitaki KD, Mountain DC (2007) Imaging electrically evoked micromechanical motion within the organ of corti of the excised gerbil cochlea. Biophys J 92:3294-3316.

Kazmierczak P, Sakaguchi H, Tokita J, Wilson-Kubalek EM, Milligan RA, Müller U, Kachar B (2007) Cadherin 23 and protocadherin 15 interact to form tip-link filaments in sensory hair cells. Nature 449:87-91.

Kozlov AS, Risler T, Hudspeth AJ (2007) Coherent motion of stereocilia assures the concerted gating of hair-cell transduction channels. Nat Neurosci 10:87-92.
Martin P, Mehta AD, Hudspeth AJ (2000) Negative hair-bundle stiffness betrays a mechanism for mechanical amplification by the hair cell. Proc Natl Acad Sci U S A 97:12026-12031.

Meyers JR, MacDonald RB, Duggan A, Lenzi D, Standaert DG, Corwin JT, Corey DP (2003) Lighting up the senses: FM1-43 loading of sensory cells through nonselective ion channels. J Neurosci 23:4054-4065.

Nagel G, Neugebauer D-C, Schmidt B, Thurm U (1991) Structures transmitting stimulatory force to the sensory hairs of vestibular ampullae of fishes and frog. Cell Tissue Res 265:567-578.

Nam JH, Fettiplace R (2008) Theoretical conditions for high-frequency hair bundle oscillations in auditory hair cells. Biophys J 95:4948-4962.

Nam JH, Cotton JR, Peterson EH, Grant W (2006) Mechanical properties and consequences of stereocilia and extracellular links in vestibular hair bundles. Biophys J 90:2786-2795.

Nam JH, Cotton JR, Grant W (2007a) A virtual hair cell, I: Addition of gating spring theory into a 3-D bundle mechanical model. Biophys J 92:1918-1928.

Nam JH, Cotton JR, Grant W (2007b) A virtual hair cell, II: Evaluation of mechanoelectric transduction parameters. Biophys J 92:1929-1937.

Nayak GD, Ratnayaka HS, Goodyear RJ, Richardson GP (2007) Development of the hair bundle and mechanotransduction. Int J Dev Biol 51:597-608.

Neugebauer D-C, Thurm U (1987) Surface charges of the membrane and cell adhesion substances determine the structural integrity of hair bundles from the inner ear of fish. Cell Tissue Res 249:199-207.

Pickles JO (1993) A model for the mechanics of the stereociliar bundle on acousticolateral hair cells. Hear Res 68:159-172.

Pickles JO, Comis SD, Osborne MP (1984) Cross-links between stereocilia in the guinea pig organ of Corti, and their possible relation to sensory transduction. Hear Res 15:103-112.

Santi PA, Anderson CB (1987) A newly identified surface coat on cochlear hair cells. Hear Res 27:47-65.

Sathyanarayana BK, Hahn Y, Patankar MS, Pastan I, Lee B (2009) Mesothelin, stereocilin, and otoancorin are predicted to have superhelical structures with ARM-type repeats. BMC Struct Biol 9:1.

Shepherd GM, Corey DP, Block SM (1990) Actin cores of hair-cell stereocilia support myosin motility. Proc Natl Acad Sci U S A 87:8627-8631.

Shi X, Gillespie PG, Nuttall AL (2005) $\mathrm{Na}^{+}$influx triggers bleb formation on inner hair cells. Am J Physiol Cell Physiol 288:C1332-C1341.

Sotomayor M, Weihofen WA, Gaudet R, Corey DP (2010) Structural determinants of cadherin-23 function in hearing and deafness. Neuron 66:85-100.

Tinevez JY, Jülicher F, Martin P (2007) Unifying the various incarnations of active hair-bundle motility by the vertebrate hair cell. Biophys J 93:4053-4067.

Tsuprun V, Santi P (1998) Structure of outer hair cell stereocilia links in the chinchilla. J Neurocytol 27:517-528.

Tsuprun V, Santi P (2002) Structure of outer hair cell stereocilia side and attachment links in the chinchilla cochlea. J Histochem Cytochem 50:493-502.

Tsuprun V, Schachern PA, Cureoglu S, Paparella M (2003) Structure of the stereocilia side links and morphology of auditory hair bundle in relation to noise exposure in the chinchilla. J Neurocytol 32:1117-1128.

Tsuprun V, Goodyear RJ, Richardson GP (2004) The structure of tip links and kinocilial links in avian sensory hair bundles. Biophys J 87:4106-4112.

van Benthem PP, de Groot JC, Albers FW, Veldman JE, Huizing EH (1993) Structure and composition of stereocilia cross-links in normal and hydropic cochleas of the guinea pig. Eur Arch Otorhinolaryngol 250:73-77.

Verpy E, Masmoudi S, Zwaenepoel I, Leibovici M, Hutchin TP, Del Castillo I, Nouaille S, Blanchard S, Lainé S, Popot JL, Moreno F, Mueller RF, Petit C (2001) Mutations in a new gene encoding a protein of the hair bundle cause non-syndromic deafness at the DFNB16 locus. Nat Genet 29:345-349.

Verpy E, Weil D, Leibovici M, Goodyear RJ, Hamard G, Houdon C, Lefèvre GM, Hardelin JP, Richardson GP, Avan P, Petit C (2008) Stereocilindeficient mice reveal the origin of cochlear waveform distortions. Nature 456:255-258.

Weiss TF, Leong R (1985) A model for signal transmission in an ear having hair cells with free-standing stereocilia. III. Micromechanical stage. Hear Res 20:157-174.

Zetes DE, Steele CR (1997) Fluid-structure interaction of the stereocilia bundle in relation to mechanotransduction. J Acoust Soc Am 101:3593-3601. 